\title{
Synthesis and Reactivity of New Copper(I) Hydride Dimers
}

\author{
Abraham J. Jordan, ${ }^{\dagger}$ Chelsea M. Wyss, ${ }^{\dagger}$ John Bacsa, ${ }^{\star}$ Joseph P. Sadighi, ${ }^{*}, \dagger$ \\ ${ }^{\dagger}$ School of Chemistry and Biochemistry, Georgia Institute of Technology, Atlanta, Georgia 30332- \\ 0400, United States \\ $\$$ X-ray Crystallography Center, Department of Chemistry, Emory University, 1515 Dickey Drive, \\ Atlanta, Georgia 30322, United States \\ joseph.sadighi@chemistry.gatech.edu
}

Supporting Information

\section{Contents}

General Considerations

Spectroscopic Measurements

S-3

Experimental Procedures

Reaction of $[(5 \mathrm{Dipp}) \mathrm{CuH}]_{2}$ with 1-Hexene

S-26

Reaction of $[(7 \mathrm{Dipp}) \mathrm{CuH}]_{2}$ with benzyl isonitrile

X-Ray Diffraction Studies

References

S-40 
General Considerations. Unless otherwise indicated, manipulations were performed in an MBraun glovebox under an inert atmosphere of nitrogen, or in sealable glassware on a Schlenk line under an atmosphere of argon. Glassware and magnetic stir bars were dried in a ventilated oven at $160^{\circ} \mathrm{C}$ and were allowed to cool under vacuum. Dichloromethane (BDH), hexane (EMD Millipore Omnisolv), tetrahydrofuran (THF, EMD Millipore Omnisolv), toluene (EMD Millipore Omnisolv) were sparged with ultra high purity argon (NexAir) for 30 minutes prior to first use, dried using an MBraun solvent purification system, transferred to Straus flasks, degassed using three freeze-pumpthaw cycles, and stored under nitrogen or argon. Anhydrous benzene (EMD Millipore Drisolv) and, anhydrous pentane (EMD Millipore Drisolv, sealed under a nitrogen atmosphere) were used as received and stored in a glovebox. Tap water was purified in a Barnstead International automated still prior to use.

Benzene- $d_{6}$ (Cambridge Isotope Labs) was dried over sodium benzophenone ketyl, vacuumtransferred into oven-dried resealable flasks, and degassed by successive freeze-pump-thaw cycles. Dichloromethane- $d_{2}$ (Cambridge Isotope Laboratories) was dried over calcium hydride overnight, vacuum-transferred to an oven-dried resealable Schlenk flask, and degassed by successive freezepump-thaw cycles.

Sodium tert-butoxide (TCI America), copper(I) chloride (Alfa-Aesar), 4,4,5,5-tetramethyl1,3,2-dioxaborolane (Sigma-Aldrich), benzyl isonitrile (Sigma-Aldrich), 2,6-diisopropylaniline (Sigma-Aldrich), N,N-diisopropylethylamine (Alfa-Aesar), acetic acid, (Alfa-Aesar), sodium metal (Alfa-Aesar), benzophenone (Alfa-Aesar), 1,2-dichloroethane (EMD Millipore Omnisolv), 1,3dichloropropane (TCI America), 1,4-dibromobutane (Sigma-Aldrich) 1,4- triethylorthoformate (Alfa-Aesar), Sodium bis(trimethylsilyl)amide (NaHMDS, Sigma-Aldrich), nitrogen (NexAir), and argon (both industrial and ultra-high purity grades, NexAir) were used as received. Carbon dioxide (NexAir) was passed through $\mathrm{P}_{2} \mathrm{O}_{5}$ prior to use. 1-Hexene (Alfa-Aesar) was filtered over alumina (EMD) prior to use. 5Dipp $\cdot \mathrm{HCl}^{1},{ }^{1}$ (5Dipp) $\mathrm{CuCl}^{2},{ }^{2}$ and (5Dipp)CuOtBu, ${ }^{2} N, N^{\prime}$-bis(2,6-diisopropyl- 
phenyl)formamidine, ${ }^{3}$ and $7 \mathrm{Dipp} \cdot \mathrm{HBF}_{4}{ }^{3}$ were prepared according to literature protocols and were characterized by ${ }^{1} \mathrm{H}$ NMR spectroscopy.

Spectroscopic Measurements. ${ }^{1} \mathrm{H}$ and ${ }^{13} \mathrm{C}$ spectra were obtained using a Varian Vx 400 $\mathrm{MHz}$ spectrometer. ${ }^{1} \mathrm{H}$ and ${ }^{13} \mathrm{C}$ NMR chemical shifts are referenced with respect to solvent signals and reported relative to tetramethylsilane. Unless otherwise stated, infrared spectra were collected using microcrystalline samples on a Bruker Alpha-P infrared spectrometer equipped with an attenuated total reflection (ATR) attachment. Samples were exposed to air as briefly as possible prior to data collection. UV-visible absorption spectra were acquired using a Varian Cary 50 spectrophotometer. Unless otherwise noted, all electronic absorption spectra were recorded at ambient temperatures in $1 \mathrm{~cm}$ quartz cells.

Elemental analyses were performed by Atlantic Microlab, Inc. in Norcross, Georgia. 


\section{Experimental Procedures}

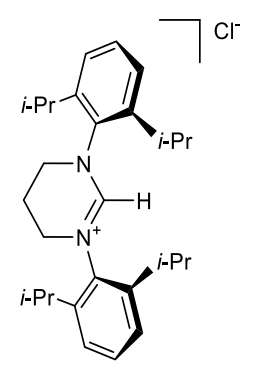

6Dipp•HCl. $N, N$-Diisopropylethylamine $(1.95 \mathrm{~mL}, 15.0 \mathrm{mmol})$ was added to a mixture of $N, N$ bis(2,6-diisopropylphenyl)formamidine $(5.0 \mathrm{~g}, 14 \mathrm{mmol})$, and 1,3-dichloropropane (6.5 mL, 69 mmol) This was heated to $120^{\circ} \mathrm{C}$, equipped with a reflux condenser and allowed to stir for $16 \mathrm{~h}$. Excess 1,3-dichloropropane was then removed in vacuo. The resulting brown solid was dissolved in $\mathrm{CH}_{2} \mathrm{Cl}_{2}(20 \mathrm{~mL})$, washed with saturated aqueous $\mathrm{K}_{2} \mathrm{CO}_{3}(3 \times 10 \mathrm{~mL})$ and $\mathrm{H}_{2} \mathrm{O}(1 \times 10 \mathrm{~mL})$, dried over $\mathrm{MgSO}_{4}$, and concentrated in vacuo. This resulting brown solid was dissolved in $\mathrm{CH}_{2} \mathrm{Cl}_{2}(30$ $\mathrm{mL})$ and toluene $(10 \mathrm{~mL})$ before the $\mathrm{CH}_{2} \mathrm{Cl}_{2}$ was removed in vacuo. The resulting in a colorless precipitate was collected on a frit, washed with 2 portions of toluene $(5 \mathrm{~mL})$, and dried overnight under vacuum to give a colorless solid. (4.06g, 67\%). ${ }^{1} \mathrm{H}$ NMR (400 MHz, $\left.\mathrm{CDCl}_{3}\right): \delta(\mathrm{ppm}) 7.54$ (s, $1 \mathrm{H}, \mathrm{NC}(H) \mathrm{N}), 7.43\left(\mathrm{t},{ }^{3} J_{\mathrm{HH}}=7.6 \mathrm{~Hz}, 2 \mathrm{H}\right.$, para-CH), $7.25\left(\mathrm{~d},{ }^{3} J_{\mathrm{HH}}=7.8 \mathrm{~Hz}, 4 \mathrm{H}\right.$, meta-CH$), 4.22(\mathrm{t}$, $\left.{ }^{3} J_{\mathrm{HH}}=5.6 \mathrm{~Hz}, 4 \mathrm{H}, \mathrm{NCH} H_{2}\right), 3.01\left(\mathrm{sept},{ }^{3} \mathrm{~J}_{\mathrm{HH}}=6.8 \mathrm{~Hz}, 4 \mathrm{H}, \mathrm{CH}\left(\mathrm{CH}_{3}\right)_{2}\right), 2.81\left(\mathrm{~m}, 2 \mathrm{H}, \mathrm{NCH}_{2} \mathrm{CH}_{2}\right)$, $1.37\left(\mathrm{~d},{ }^{3} J_{\mathrm{HH}}=6.8 \mathrm{~Hz}, 12 \mathrm{H}, \mathrm{CH}_{2}\left(\mathrm{CH}_{3}\right)_{2}\right), 1.22\left(\mathrm{~d},{ }^{3} J_{\mathrm{HH}}=6.8 \mathrm{~Hz}, 12 \mathrm{H}, \mathrm{CH}_{2}\left(\mathrm{CH}_{3}\right)_{2}\right){ }^{13} \mathrm{C}\left\{{ }^{1} \mathrm{H}\right\} \mathrm{NMR}$ $\left(100 \mathrm{MHz}, \mathrm{CDCl}_{3}\right): \delta(\mathrm{ppm}) 152.9(\mathrm{NC}(H) \mathrm{N}), 145.3($ ortho-C), 135.5 (ipso-C), 131.2 (meta-C), 125.0 (para-C), $48.9\left(\mathrm{NCH}_{2}\right), 28.8\left(\mathrm{CH}\left(\mathrm{CH}_{3}\right)_{2}\right), 24.7\left(\mathrm{CH}\left(\mathrm{CH}_{3}\right)_{2}\right), 24.7\left(\mathrm{CH}\left(\mathrm{CH}_{3}\right)_{2}\right), 19.2$ $\left(\mathrm{NCH}_{2} \mathrm{CH}_{2}\right)$.

Note: This is a previously reported compound that was prepared via a different synthetic route. ${ }^{4}$ 


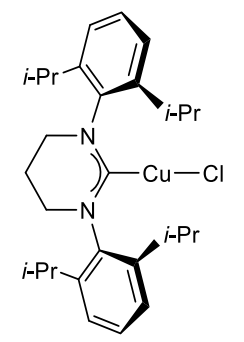

(6Dipp)CuCl. NaHMDS (0.411 g, $2.25 \mathrm{mmol})$ was added to a solution of 6Dipp•HCl (0.972 g, 2.20 $\mathrm{mmol})$ in THF $(10 \mathrm{~mL})$ with stirring. After $2 \mathrm{~h}, \mathrm{CuCl}(0.222 \mathrm{~g}, 2.25 \mathrm{mmol})$ was added and the mixture was allowed to stir overnight before being filtered through Celite, washed with $\mathrm{CH}_{2} \mathrm{Cl}_{2}$ and concentrated in vacuo to give a colorless solid. This was dissolved in a minimal amount of $\mathrm{CH}_{2} \mathrm{Cl}_{2}$, layered with hexanes and placed in the freezer overnight. The resulting colorless solid was collected on a frit and, dried in vacuo to afford the title complex. (0.920 g, $83 \%) .{ }^{1} \mathrm{H}$ NMR (400 MHz, $\left.\mathrm{CDCl}_{3}\right): \delta(\mathrm{ppm}) 7.36\left(\mathrm{t},{ }^{3} J_{\mathrm{HH}}=7.6 \mathrm{~Hz}, 2 \mathrm{H}\right.$, para-CH), $7.21\left(\mathrm{~d},{ }^{3} J_{\mathrm{HH}}=7.6 \mathrm{~Hz}, 4 \mathrm{H}\right.$, meta $\left.-\mathrm{CH}\right), 3.43$ $\left(\mathrm{t},{ }^{3} J_{\mathrm{HH}}=6.0 \mathrm{~Hz}, 4 \mathrm{H}, \mathrm{NCH}{ }_{2}\right), 3.06\left(\mathrm{sept},{ }^{3} J_{\mathrm{HH}}=6.8 \mathrm{~Hz}, 4 \mathrm{H}, \mathrm{CH}\left(\mathrm{CH}_{3}\right)_{2}\right), 2.37$ (quin, ${ }^{3} J_{\mathrm{HH}}=6.0 \mathrm{~Hz}$, $\left.2 \mathrm{H}, \mathrm{NCH}_{2} \mathrm{CH}_{2}\right), 1.34\left(\mathrm{~d},{ }^{3} J_{\mathrm{HH}}=7.2 \mathrm{~Hz}, 12 \mathrm{H}, \mathrm{CH}_{2}\left(\mathrm{CH}_{3}\right)_{2}\right), 1.31\left(\mathrm{~d},{ }^{3} J_{\mathrm{HH}}=7.2 \mathrm{~Hz}, 12 \mathrm{H}, \mathrm{CH}_{2}\left(\mathrm{CH}_{3}\right)_{2}\right)$. ${ }^{13} \mathrm{C}\left\{{ }^{1} \mathrm{H}\right\}$ NMR $\left(100 \mathrm{MHz}, \mathrm{CDCl}_{3}\right): \delta(\mathrm{ppm}) 200.8(\mathrm{NCCu}), 145.6$ (ortho-C), 141.5 (ipso-C), 129.5 (meta-C), 124.8 (para-C), $46.3\left(\mathrm{NCH}_{2}\right), 28.8\left(\mathrm{CH}\left(\mathrm{CH}_{3}\right)_{2}\right), 25.0\left(\mathrm{CH}\left(\mathrm{CH}_{3}\right)_{2}\right), 24.8\left(\mathrm{CH}\left(\mathrm{CH}_{3}\right)_{2}\right), 20.6$ $\left(\mathrm{NCH}_{2} \mathrm{CH}_{2}\right)$.

Note: This is a previously reported compound that was prepared via a different synthetic route. ${ }^{5}$ 


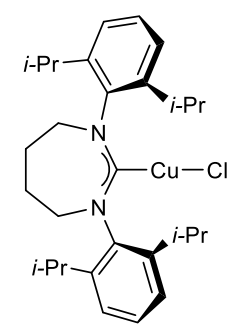

(7Dipp)CuCl NaHMDS (0.379 g, $2.07 \mathrm{mmol})$ was added to a solution of 7Dipp $\cdot \mathrm{HBF}_{4}(1.00 \mathrm{~g}, 1.97$ $\mathrm{mmol})$ in THF $(10 \mathrm{~mL})$ with stirring. After $2 \mathrm{~h}, \mathrm{CuCl}(0.207 \mathrm{~g}, 2.09 \mathrm{mmol})$ was added and the mixture was allowed to stir overnight before being filtered through Celite, washed with $\mathrm{CH}_{2} \mathrm{Cl}_{2}$ and concentrated in vacuo to give colorless solid. This was dissolved in a minimal amount of $\mathrm{CH}_{2} \mathrm{Cl}_{2}$, layered with hexanes and placed in the freezer overnight. The resulting colorless solid was collected on a frit and, dried in vacuo to afford the title complex. $(0.804 \mathrm{~g}, 79 \%) .{ }^{1} \mathrm{H}$ NMR $\left(400 \mathrm{MHz}, \mathrm{C}_{6} \mathrm{D}_{6}\right)$ : $\delta(\mathrm{ppm}) 7.32\left(\mathrm{t},{ }^{3} J_{\mathrm{HH}}=7.6 \mathrm{~Hz}, 2 \mathrm{H}\right.$, para-CH), $7.18\left(\mathrm{~d},{ }^{3} J_{\mathrm{HH}}=7.6 \mathrm{~Hz}, 4 \mathrm{H}\right.$, meta-CH), $3.97(\mathrm{~m}, 4 \mathrm{H}$, $\left.\mathrm{NCH}_{2}\right), 3.25\left(\mathrm{sept},{ }^{3} J_{\mathrm{HH}}=6.8 \mathrm{~Hz}, 4 \mathrm{H}, \mathrm{CH}\left(\mathrm{CH}_{3}\right)_{2}\right), 2.33\left(\mathrm{~m}, 4 \mathrm{H}, \mathrm{NCH}_{2} \mathrm{CH}_{2}\right), 1.35\left(\mathrm{~d},{ }^{3} J_{\mathrm{HH}}=6.8 \mathrm{~Hz}\right.$, $\left.12 \mathrm{H}, \mathrm{CH}_{2}\left(\mathrm{CH}_{3}\right)_{2}\right), 1.32\left(\mathrm{~d},{ }^{3} \mathrm{~J}_{\mathrm{HH}}=6.8 \mathrm{~Hz}, 12 \mathrm{H}, \mathrm{CH}_{2}\left(\mathrm{CH}_{3}\right)_{2}\right) .{ }^{13} \mathrm{C}\left\{{ }^{1} \mathrm{H}\right\} \mathrm{NMR}\left(100 \mathrm{MHz}, \mathrm{C}_{6} \mathrm{D}_{6}\right): \delta$ (ppm) $210.3(\mathrm{NCCu}), 145.0$ (ortho-C), 144.0 (ipso-C), 129.1 (meta-C), 124.9 (para-C), 54.0 $\left(\mathrm{NCH}_{2}\right), 28.9\left(\mathrm{CH}\left(\mathrm{CH}_{3}\right)_{2}\right), 25.3\left(\mathrm{NCH}_{2} \mathrm{CH}_{2}\right), 24.8\left(\mathrm{CH}\left(\mathrm{CH}_{3}\right)_{2}\right), 24.8\left(\mathrm{CH}\left(\mathrm{CH}_{3}\right)_{2}\right), \mathrm{IR}: v\left(\mathrm{~cm}^{-1}\right) 2960$, 2873, 1496, 1447, 1385, 1362, 1311, 1290, 1177, 1056, 936, 904, 804, 785, 761, 573, 450 Anal. Calcd for $\mathrm{C}_{29} \mathrm{H}_{42} \mathrm{ClCuN}_{2}$ : C, 67.29; H, 8.18; N, 5.41. Found C, 67.35; H, 8.23; N, 5.42. 


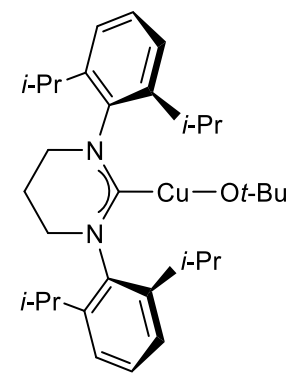

(6Dipp)Cu(Ot-Bu). Sodium tert-butoxide $(0.033 \mathrm{~g}, 0.34 \mathrm{mmol})$ was added to a solution of (6Dipp) $\mathrm{CuCl}(0.170 \mathrm{~g}, 0.34 \mathrm{mmol})$ in THF $(4 \mathrm{~mL})$ with stirring. After 3 hours, the reaction mixture was filtered through Celite, and the filter pad was washed with 2 portions of THF ( $1 \mathrm{~mL}$ each). The filtrate was concentrated, and the residue was dried for 12 hours at $40^{\circ} \mathrm{C}$ under vacuum, affording the product at a colorless powder $(0.174 \mathrm{~g}, 96 \%) .{ }^{1} \mathrm{H}$ NMR $\left(400 \mathrm{MHz}, \mathrm{C}_{6} \mathrm{D}_{6}\right): \delta(\mathrm{ppm}) 7.13\left(\mathrm{dd},{ }^{3} J_{\mathrm{HH}}\right.$ $=8.4,6.8 \mathrm{~Hz}, 2 \mathrm{H}$ para-CH), $7.05\left(\mathrm{~d},{ }^{3} J_{\mathrm{HH}}=7.2 \mathrm{~Hz}, 4 \mathrm{H}\right.$, meta-CH), $3.03\left(\right.$ sept, ${ }^{3} J_{\mathrm{HH}}=6.8 \mathrm{~Hz}, 4 \mathrm{H}$, $\left.\left.\mathrm{C} H\left(\mathrm{CH}_{3}\right)_{2}\right), 2.68(\mathrm{t}, J=6.0 \mathrm{~Hz}, 4 \mathrm{H}, \mathrm{NCH})_{2}\right), 1.57\left(\mathrm{~d},{ }^{3} J_{\mathrm{HH}}=7.2 \mathrm{~Hz}, 12 \mathrm{H}, \mathrm{CH}_{2}\left(\mathrm{CH}_{3}\right), 1.44\right.$ (quin, ${ }^{3} J_{\mathrm{HH}}$ $\left.=6.0 \mathrm{~Hz}, 2 \mathrm{H}, \mathrm{NCH}_{2} \mathrm{CH}_{2}\right), 1.18\left(\mathrm{~d},{ }^{3} J_{\mathrm{HH}}=7.2 \mathrm{~Hz}, 12 \mathrm{H}, \mathrm{CH}_{2}\left(\mathrm{CH}_{3}\right), 1.16\left(\mathrm{~s}, 9 \mathrm{H}, \mathrm{C}\left(\mathrm{CH}_{3}\right)_{3}\right) .{ }^{13} \mathrm{C}\left\{{ }^{1} \mathrm{H}\right\}\right.$ NMR (100 MHz, $\left.\mathrm{C}_{6} \mathrm{D}_{6}\right): \delta(\mathrm{ppm}) 203.5(\mathrm{NCCu}), 145.5$ (ortho-C), 142.6 (ipso-C), 129.3 (meta-C),

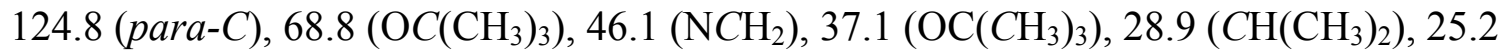
$\left(\mathrm{CH}\left(\mathrm{CH}_{3}\right)_{2}\right), 24.8\left(\mathrm{CH}\left(\mathrm{CH}_{3}\right)_{2}\right), 20.4\left(\mathrm{NCH}_{2} \mathrm{CH}_{2}\right)$. IR: $v\left(\mathrm{~cm}^{-1}\right)$ 2957, 2869, 1492, 1473, 1385, 1363 , 1323, 1307, 1291, 1195, 1094, 1057, 970, 803, 787, 760, 592, 551, 457 Anal. Calcd for $\mathrm{C}_{32} \mathrm{H}_{49} \mathrm{CuN}_{2} \mathrm{O}$ : C, 71.00; H, 9.12; N, 5.18. Found C, 69.74; H, 9.19; N, 5.10. 


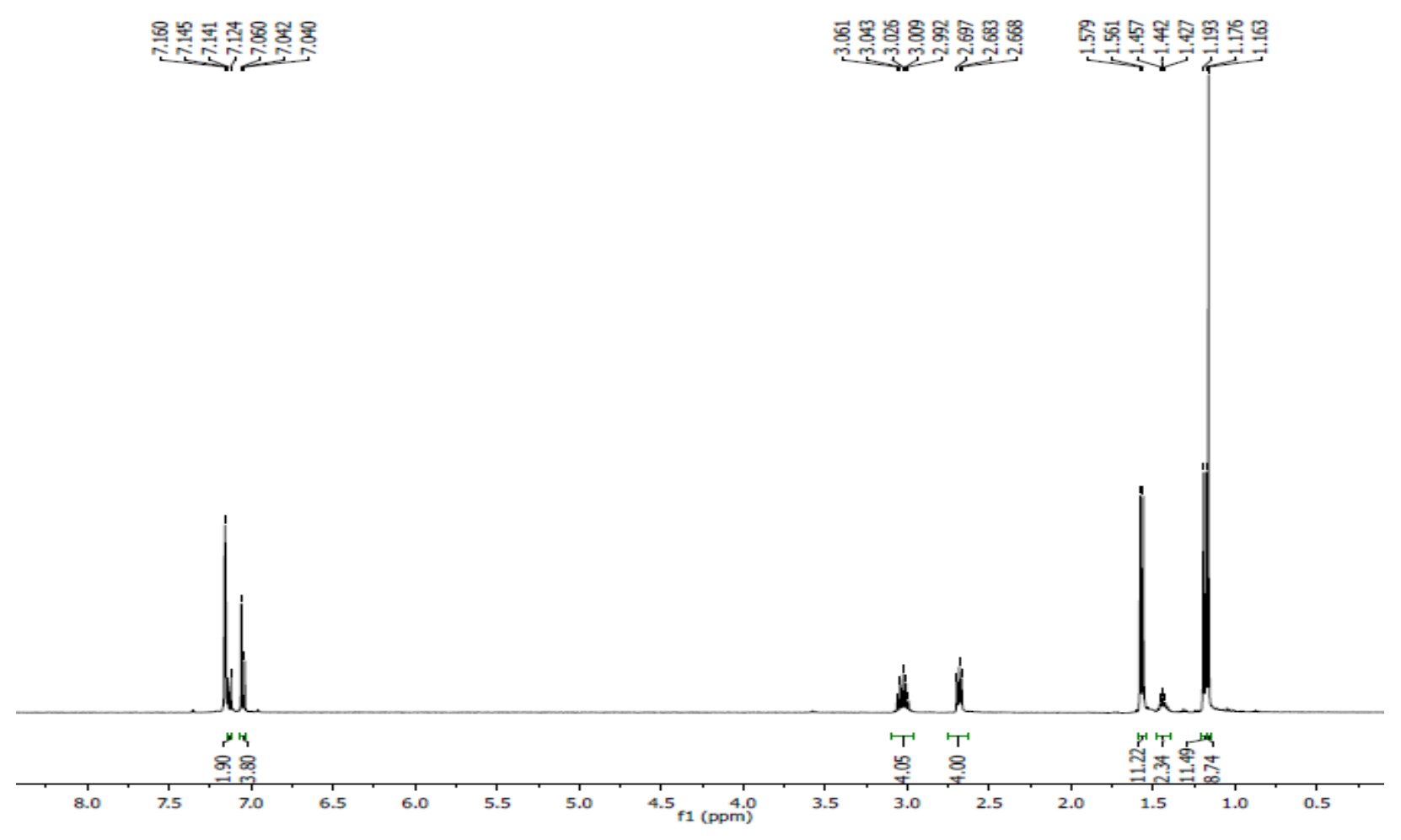

Figure S1. ${ }^{1} \mathrm{H}$ NMR spectrum of $6 \mathrm{DippCuOt}$ - $\mathrm{Bu}$ in $\mathrm{C}_{6} \mathrm{D}_{6}$.

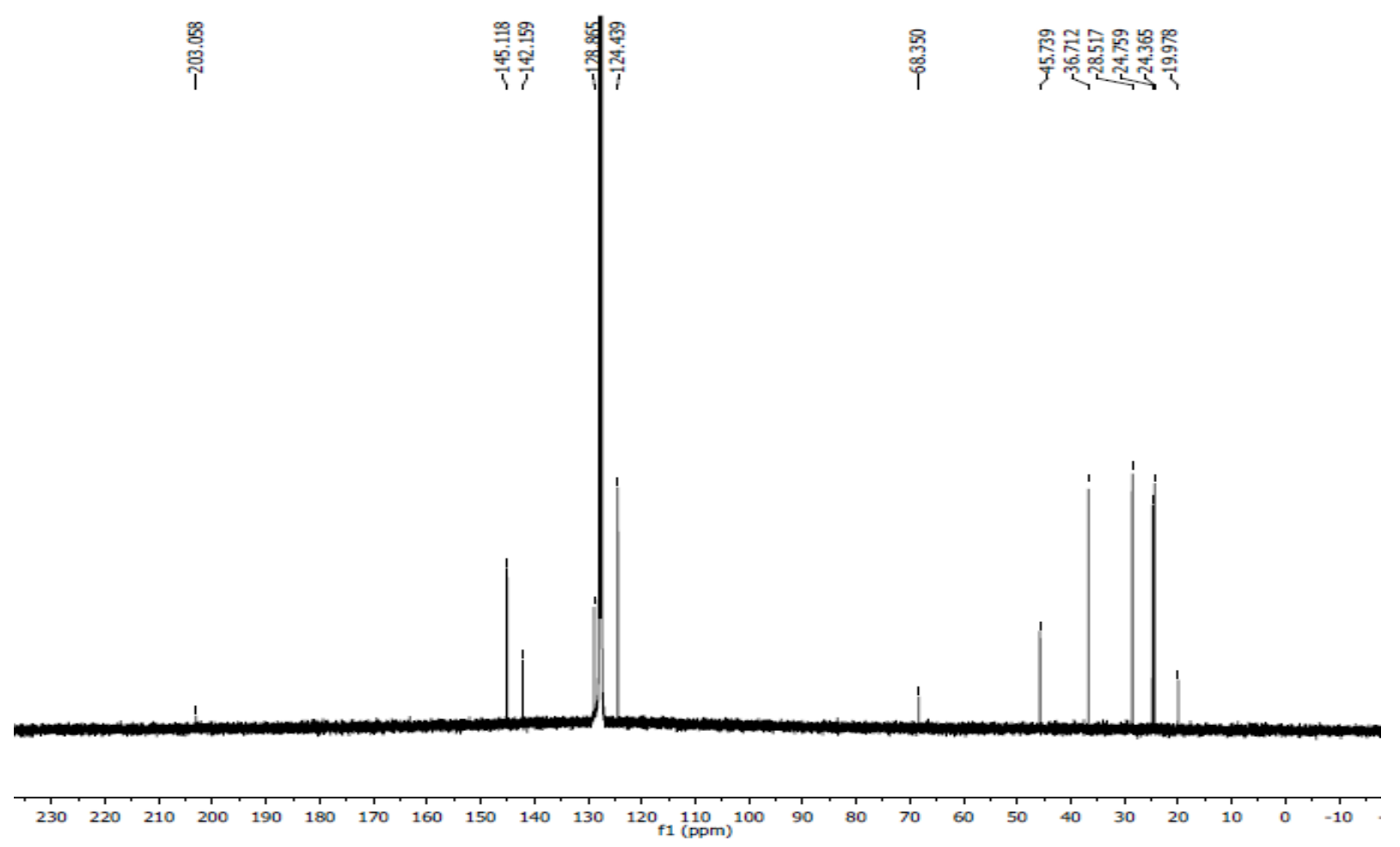

Figure S2. ${ }^{13} \mathrm{C}$ NMR spectrum of $6 \mathrm{DippCuOt} t-\mathrm{Bu}$ in $\mathrm{C}_{6} \mathrm{D}_{6}$. 


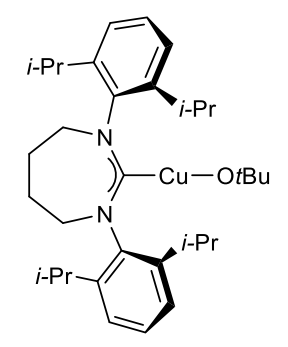

(7Dipp)Cu(Ot-Bu). Sodium tert-butoxide $(0.059 \mathrm{~g}, 0.61 \mathrm{mmol})$ was added to a solution of (7Dipp) $\mathrm{CuCl}(0.313 \mathrm{~g}, 0.61 \mathrm{mmol})$ in THF $(8 \mathrm{~mL})$ with stirring. After 3 hours, the reaction mixture was filtered through Celite, and the filter pad was washed with 2 portions of THF ( $2 \mathrm{~mL}$ each). The filtrate was concentrated, and the residue was dried for 12 hours at $40^{\circ} \mathrm{C}$ under vacuum, affording the product at a colorless powder. $(0.272 \mathrm{~g}, 81 \%){ }^{1} \mathrm{H}$ NMR $\left(400 \mathrm{MHz}, \mathrm{C}_{6} \mathrm{D}_{6}\right): \delta(\mathrm{ppm}) 7.13\left(\mathrm{dd},{ }^{3} J_{\mathrm{HH}}\right.$ $=8.8,7.2 \mathrm{~Hz}, 2 \mathrm{H}$, para- $\mathrm{CH}), 7.05\left(\mathrm{~d},{ }^{3} J_{\mathrm{HH}}=7.6 \mathrm{~Hz}, 4 \mathrm{H}\right.$, meta $\left.-\mathrm{CH}\right), 3.26(\mathrm{~m}, 4 \mathrm{H}, \mathrm{NCH}), 3.24(\mathrm{sept}$, $\left.{ }^{3} J_{\mathrm{HH}}=6.8 \mathrm{~Hz}, 4 \mathrm{H}, \mathrm{CH}\left(\mathrm{CH}_{3}\right)_{2}\right), 1.60\left(\mathrm{~m}, 16 \mathrm{H}, \mathrm{NCH}_{2} \mathrm{CH}_{2}, \mathrm{CH}_{2}\left(\mathrm{CH}_{3}\right)_{2}\right), 1.21\left(\mathrm{~d},{ }^{3} J_{\mathrm{HH}}=6.8 \mathrm{~Hz}, 12 \mathrm{H}\right.$, $\left.\mathrm{CH}_{2}\left(\mathrm{CH}_{3}\right)_{2}\right), 1.12\left(\mathrm{~s}, 9 \mathrm{H}, \mathrm{C}\left(\mathrm{CH}_{3}\right)_{3}\right){ }^{13} \mathrm{C}\left\{{ }^{1} \mathrm{H}\right\} \mathrm{NMR}\left(100 \mathrm{MHz}, \mathrm{C}_{6} \mathrm{D}_{6}\right): \delta(\mathrm{ppm}) 213.1(\mathrm{NCCu}), 145.02$

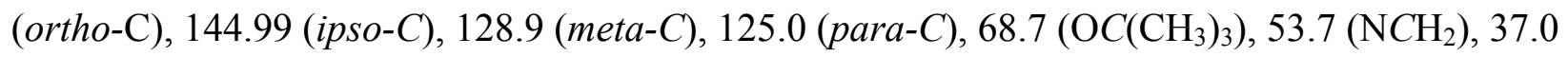
$\left(\mathrm{OC}\left(\mathrm{CH}_{3}\right)_{3}\right), 29.1\left(\mathrm{CH}\left(\mathrm{CH}_{3}\right)_{2}\right), 25.1\left(\mathrm{NCH}_{2} \mathrm{CH}_{2}\right), 24.7\left(\mathrm{CH}\left(\mathrm{CH}_{3}\right)_{2}\right), \mathrm{IR}: v\left(\mathrm{~cm}^{-1}\right)$ 2957, 1493, 1448, $1385,1363,1308,1291,1194,1094,1057,970,803,788,760,572,552,456$

Note: We have been unable to obtain satisfactory elemental analysis for (7Dipp) $\mathrm{Cu}(\mathrm{O} t-\mathrm{Bu})$. The complex is extremely moisture sensitive. While NMR silent impurities cannot be ruled out, we believe the ${ }^{1} \mathrm{H}$ and ${ }^{13} \mathrm{C}$ NMRs spectra reflect the purity of the sample 


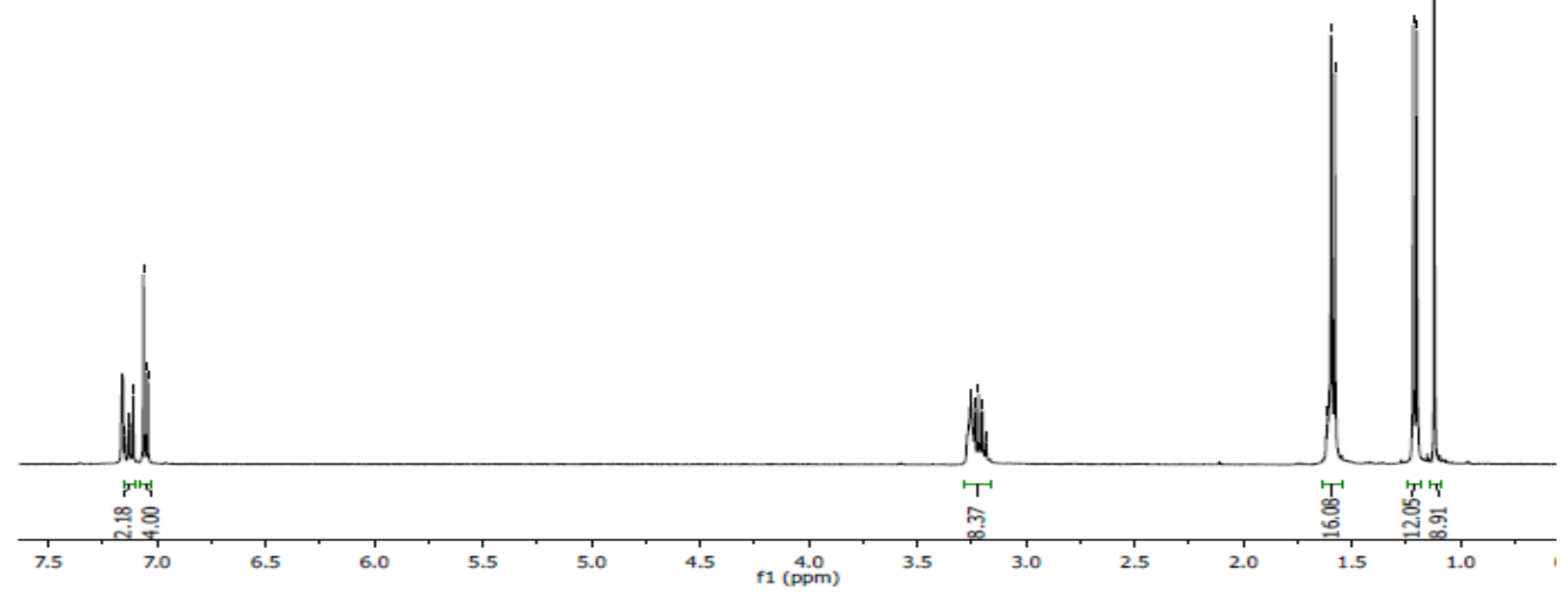

Figure S3. ${ }^{1} \mathrm{H}$ NMR spectrum of (7Dipp)CuOt-Bu in $\mathrm{C}_{6} \mathrm{D}_{6}$.
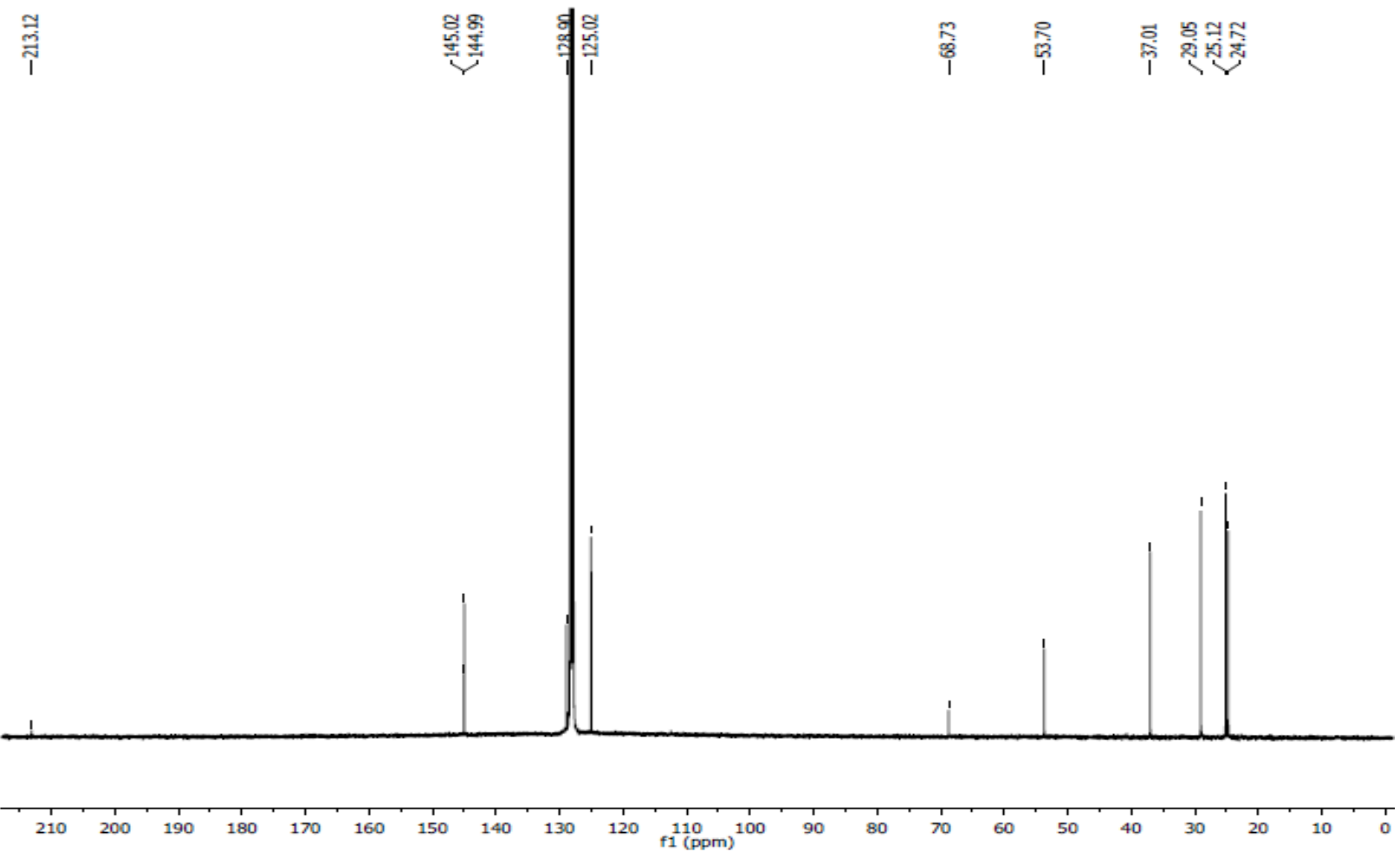

Figure S4. ${ }^{13} \mathrm{C}$ NMR spectrum of (7Dipp) $\mathrm{CuO} t$ - $\mathrm{Bu}$ in $\mathrm{C}_{6} \mathrm{D}_{6}$. 


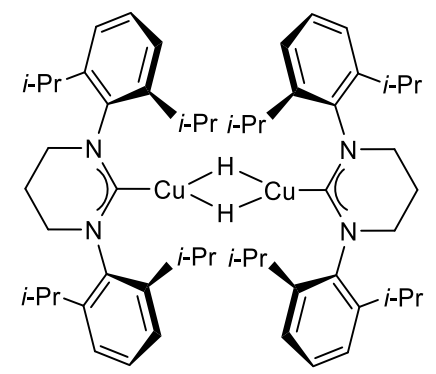

[(6Dipp)CuH] $]_{2}$ (3a) Pinacolborane 4,4,5,5,- tetramethyl-1,3,2-dioxaborolane [HB(pin)] (0.040 mL, $0.26 \mathrm{mmol})$ was added to a solution of (6Dipp) $\mathrm{Cu}(\mathrm{Ot}-\mathrm{Bu})(0.095 \mathrm{~g}, 0.18 \mathrm{mmol})$ in THF $(1 \mathrm{~mL})$ via syringe. The resulting yellow solution was layered with pentane $(2 \mathrm{~mL})$ and placed in the freezer at $-35^{\circ} \mathrm{C}$ for $12 \mathrm{~h}$. The supernatant was decanted by pipette, pentane $(1 \mathrm{~mL})$ was added and the mixture was placed back in the freezer for $4 \mathrm{~h}$. The supernatant was decanted and pentane $(1 \mathrm{~mL})$ was added again before being placed in the freezer for another $4 \mathrm{~h}$. The resulting yellow crystals were dried in vacuo for $1 \mathrm{~h}$ to afford the title complex. $(0.079 \mathrm{~g}, 96 \%) .{ }^{1} \mathrm{H}$ NMR $\left(400 \mathrm{MHz}, \mathrm{C}_{6} \mathrm{D}_{6}\right): \delta(\mathrm{ppm}) 7.21$ $\left(\mathrm{t},{ }^{3} J_{\mathrm{HH}}=7.6 \mathrm{~Hz}, 2 \mathrm{H}\right.$, para-CH), $7.00\left(\mathrm{~d},{ }^{3} J_{\mathrm{HH}}=7.6 \mathrm{~Hz}, 4 \mathrm{H}\right.$, meta-CH), $3.17\left(\mathrm{sept},{ }^{3} J_{\mathrm{HH}}=6.8 \mathrm{~Hz}\right.$, 4H, $\left.\mathrm{CH}\left(\mathrm{CH}_{3}\right)_{2}\right), 2.88\left(\mathrm{t},{ }^{3} \mathrm{~J}_{\mathrm{HH}}=6.0 \mathrm{~Hz}, 4 \mathrm{H}, \mathrm{NCH}_{2}\right.$ ), 1.65 (quin, ${ }^{3} J_{\mathrm{HH}}=6.0 \mathrm{~Hz}, 2 \mathrm{H}, \mathrm{NCH}_{2} \mathrm{CH}_{2}$ ), 1.42 $\left(\mathrm{d},{ }^{3} J_{\mathrm{HH}}=6.8 \mathrm{~Hz}, 12 \mathrm{H}, \mathrm{CH}_{2}\left(\mathrm{CH}_{3}\right)_{2}\right), 1.28\left(\mathrm{~d},{ }^{3} J_{\mathrm{HH}}=7.2 \mathrm{~Hz}, 12 \mathrm{H}, \mathrm{CH}_{2}\left(\mathrm{CH}_{3}\right)_{2}\right), 0.77(\mathrm{~s}, 1 \mathrm{H}, \mathrm{Cu}-\mathrm{H})$ ${ }^{13} \mathrm{C}\left\{{ }^{1} \mathrm{H}\right\}$ NMR (100 MHz, $\mathrm{C}_{6} \mathrm{D}_{6}$ ): $\delta(\mathrm{ppm}) 213.5$ (NCCu), 146.2 (ortho-C), 142.4 (ipso-C), 124.3 (para-C), $45.9\left(\mathrm{NCH}_{2}\right), 28.7\left(\mathrm{CH}\left(\mathrm{CH}_{3}\right)_{2}\right), 25.7\left(\mathrm{CH}\left(\mathrm{CH}_{3}\right)_{2}\right), 25.4\left(\mathrm{CH}\left(\mathrm{CH}_{3}\right)_{2}\right), 20.9\left(\mathrm{NCH}_{2} \mathrm{CH}_{2}\right)$. We believe the meta-C resonance is overlapped by the solvent signal of $\mathrm{C}_{6} \mathrm{D}_{6}$. IR: $v\left(\mathrm{~cm}^{-1}\right) 2955,2864$, $1483,1452,1384,1296,1195,1057,909,798,749,616,549,450$

Note: We have been unable to obtain satisfactory elemental analysis for $\mathbf{3 a}$. The complex is extremely air and moisture-sensitive. After exposure of a benzene solution of $\mathbf{3 a}$ to dry $\mathrm{CO}_{2}$, and concentration in vacuo, the more stable formate complex $4 \mathbf{a}$ was isolated without further purification and its elemental analysis was obtained. (see p. S-12) We reasoned that only a sufficiently pure sample of $\mathbf{3 a}$ would give rise to analytically pure $\mathbf{4 a}$. 


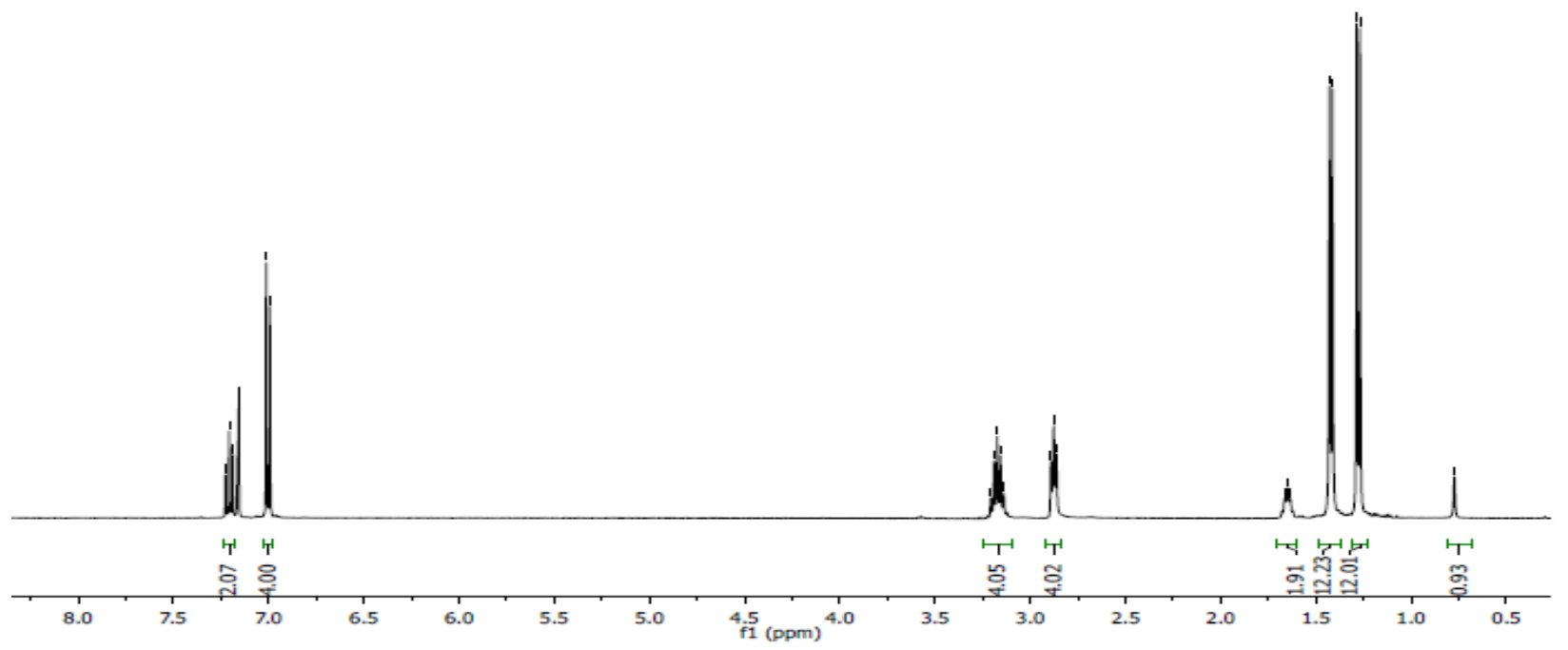

Figure S5. ${ }^{1} \mathrm{H}$ NMR spectrum of $[(6 \mathrm{Dipp}) \mathrm{CuH}]_{2}$ in $\mathrm{C}_{6} \mathrm{D}_{6}$
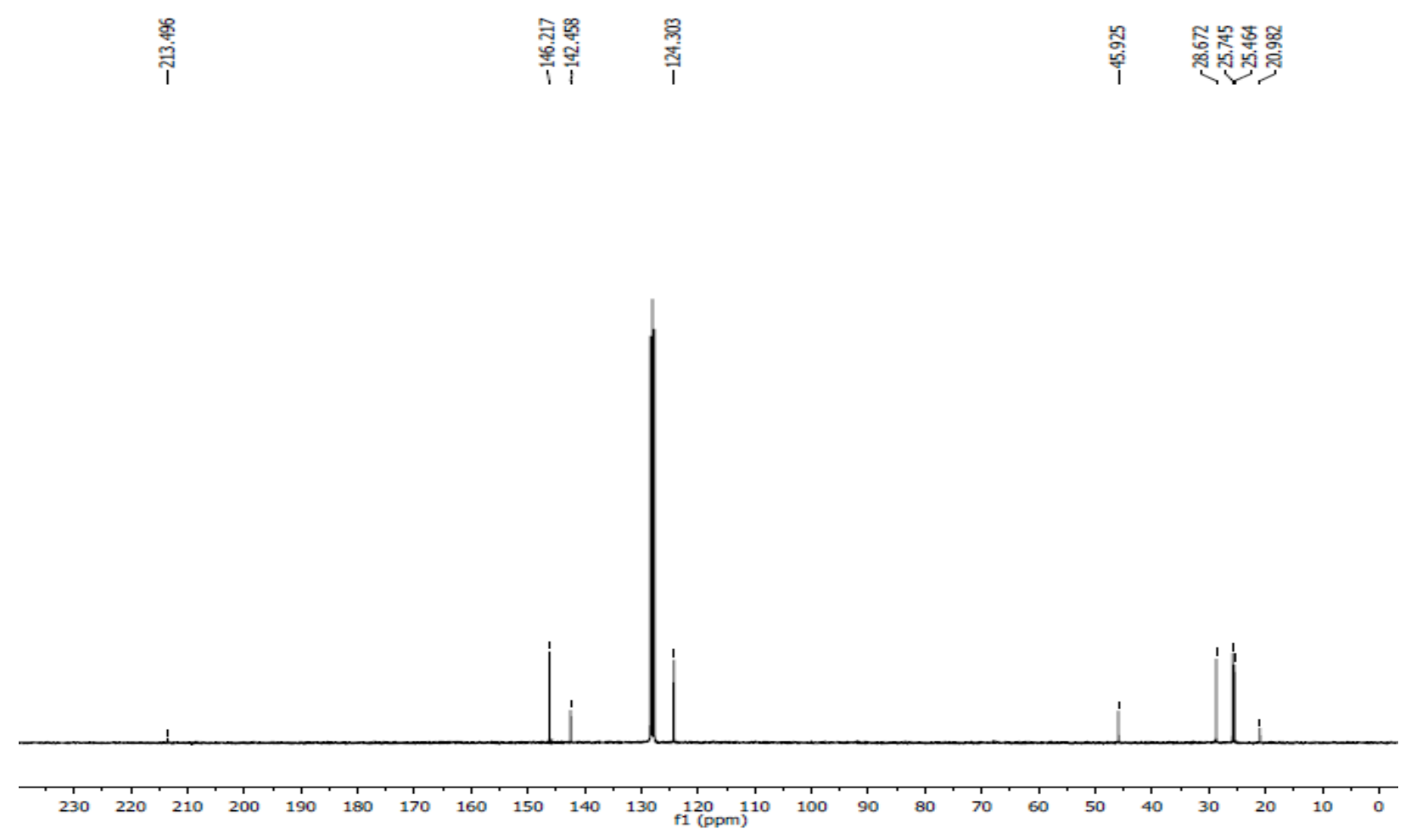

Figure S6. ${ }^{13} \mathrm{C}$ NMR spectrum of $[(6 \mathrm{Dipp}) \mathrm{CuH}]_{2}$ in $\mathrm{C}_{6} \mathrm{D}_{6}$. 
Infrared Spectra of $[(6 \mathrm{Dipp}) \mathrm{CuH}]_{2}$.

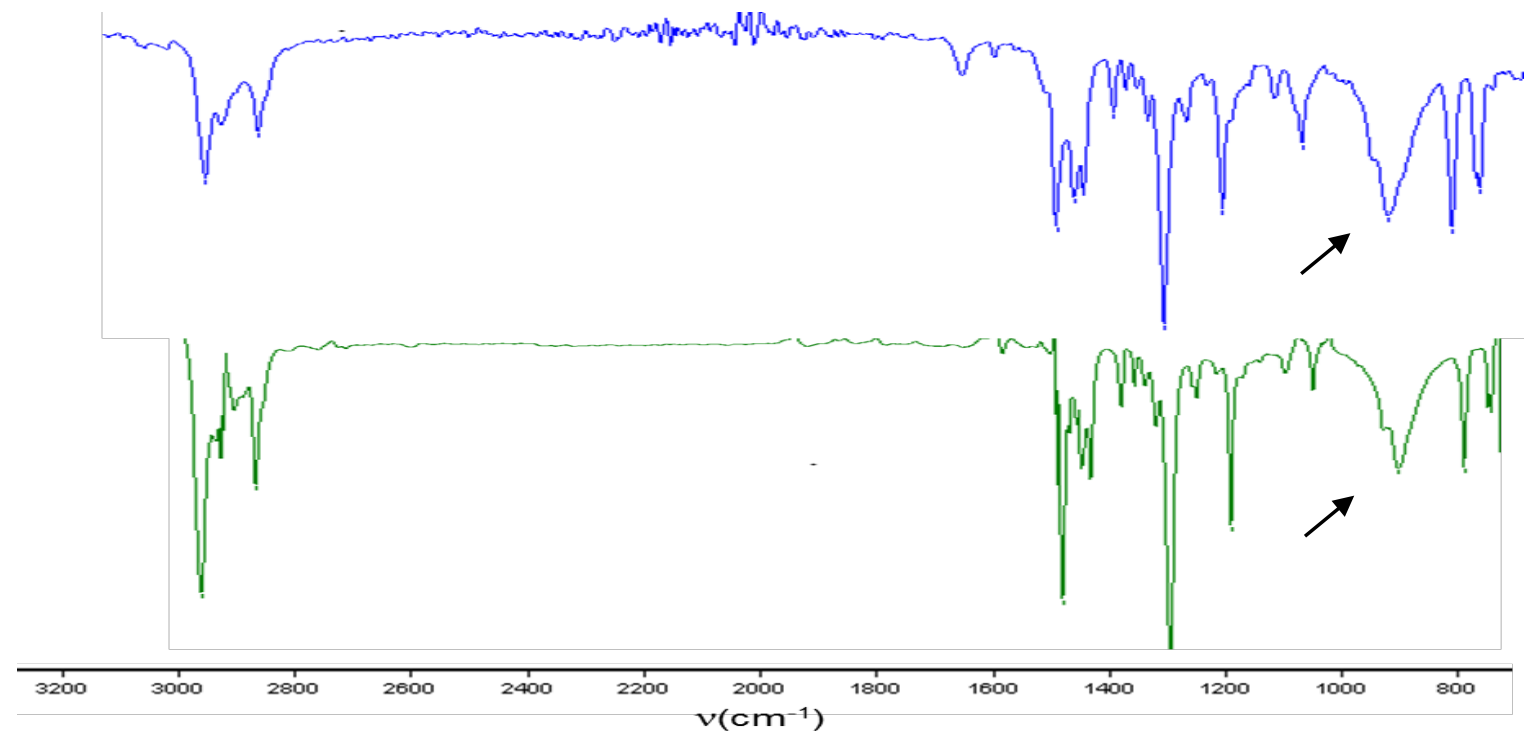

Figure S7. Infrared spectra of $[(6 \mathrm{Dipp}) \mathrm{CuH}]_{2}$, solid state (top, blue) and solution cell (bottom, green). The solution cell IR spectrum of $[(6 \mathrm{Dipp}) \mathrm{CuH}]_{2}$ was taken as a solution in toluene loaded into a $0.1 \mathrm{~mm}$ path length cell using a Bruker Alpha-P infrared spectrometer.

\section{Preparation of UV-vis absorption samples for $[(6 \mathrm{Dipp}) \mathrm{CuH}]_{2}$ and $[(7 \mathrm{Dipp}) \mathrm{CuH}]_{2}$.}

In the glovebox, $[(6 \mathrm{Dipp}) \mathrm{CuH}]_{2}(\mathbf{3 a}, 0.011 \mathrm{~g}, 0.012 \mathrm{mmol})$ was dissolved in toluene and diluted to 10 $\mathrm{mL}$ in a volumetric flask. $0.5 \mathrm{~mL}$ of this solution was diluted $10 \mathrm{~mL}$ in a separate volumetric flask before being added to a $1 \mathrm{~cm}$ sealable quartz cuvette.

In the glovebox, $[(7 \mathrm{Dipp}) \mathrm{CuH}]_{2}(\mathbf{3 b}, 0.012 \mathrm{~g}, 0.012 \mathrm{mmol})$ was dissolved in toluene and diluted to 10 $\mathrm{mL}$ in a volumetric flask. $0.5 \mathrm{~mL}$ of this solution was diluted $10 \mathrm{~mL}$ in a separate volumetric flask before being added to a $1 \mathrm{~cm}$ sealable quartz cuvette. 


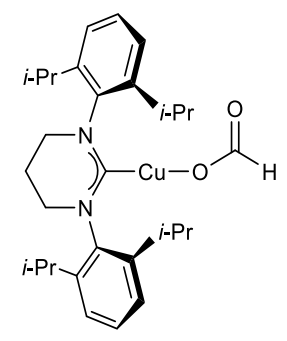

(6Dipp)copper(I) formate (4a). In an oven dried $25 \mathrm{~mL}$ Schlenk flask under $\mathrm{N}_{2}$, a solution of $[(6 \mathrm{Dipp}) \mathrm{CuH}]_{2}(0.036 \mathrm{~g}, 0.038 \mathrm{mmol})$ in $5 \mathrm{~mL}$ of benzene was degassed by 3 freeze-pump-thaw cycles before being exposed to an atmosphere of $\mathrm{CO}_{2}$. The bright yellow solution immediately became colorless, and deposited a white precipitate. The benzene was removed in vacuo and dried under vacuum for $12 \mathrm{~h}$ affording the product as a colorless powder. $(0.036 \mathrm{~g}, 94 \%){ }^{1} \mathrm{H}$ NMR $(400$ $\left.\mathrm{MHz}, \mathrm{CD}_{2} \mathrm{Cl}_{2}\right): \delta(\mathrm{ppm}) 7.68(\mathrm{~s}, 1 \mathrm{H}, \mathrm{OC}(\mathrm{O}) H) 7.39\left(\mathrm{t},{ }^{3} J_{\mathrm{HH}}=7.6 \mathrm{~Hz}, 2 \mathrm{H}\right.$, para-CH$), 7.25\left(\mathrm{~d},{ }^{3} J_{\mathrm{HH}}=\right.$ $7.6 \mathrm{~Hz}, 4 \mathrm{H}$, meta-CH), $3.43\left(\mathrm{t},{ }^{3} J_{\mathrm{HH}}=6.0 \mathrm{~Hz}, 4 \mathrm{H}, \mathrm{NCH}\right)_{2} 3.05\left(\mathrm{sept},{ }^{3} J_{\mathrm{HH}}=6.8 \mathrm{~Hz}, 4 \mathrm{H}, \mathrm{CH}\left(\mathrm{CH}_{3}\right)_{2}\right)$, 2.36 (quin, $\left.{ }^{3} J_{\mathrm{HH}}=6.0 \mathrm{~Hz}, 2 \mathrm{H}, \mathrm{NCH}_{2} \mathrm{CH}_{2}\right), 1.33\left(\mathrm{~d},{ }^{3} J_{\mathrm{HH}}=6.8 \mathrm{~Hz}, 12 \mathrm{H}, \mathrm{CH}_{2}\left(\mathrm{CH}_{3}\right)_{2}\right), 1.31\left(\mathrm{~d},{ }^{3} J_{\mathrm{HH}}=\right.$ $\left.7.2 \mathrm{~Hz}, 12 \mathrm{H}, \mathrm{CH}_{2}\left(\mathrm{CH}_{3}\right)_{2}\right){ }^{13} \mathrm{C}\left\{{ }^{1} \mathrm{H}\right\} \mathrm{NMR}\left(100 \mathrm{MHz}, \mathrm{CD}_{2} \mathrm{Cl}_{2}\right): \delta(\mathrm{ppm}) 200.6(\mathrm{NCCu}), 166.7$ $(\mathrm{OC}(\mathrm{O}) \mathrm{H}) 146.2$ (ortho-C), 142.1 (ipso-C), 129.5 (meta-C), 125.0 (para-C), $46.6\left(\mathrm{NCH}_{2}\right), 29.0$ $\left(\mathrm{CH}\left(\mathrm{CH}_{3}\right)_{2}\right), 24.9\left(\mathrm{CH}\left(\mathrm{CH}_{3}\right)_{2}\right), 24.7\left(\mathrm{CH}\left(\mathrm{CH}_{3}\right)_{2}\right), 20.8\left(\mathrm{NCH}_{2} \mathrm{CH}_{2}\right) . \mathrm{IR} v\left(\mathrm{~cm}^{-1}\right)$ 2961, 2926, 2866, $2794,1621,1509,1455,1309,1255,1206,1180,1149,1108,1078,1058,985,938,803,758,552$, 516, 445. Anal. Calcd for $\mathrm{C}_{29} \mathrm{H}_{42} \mathrm{CuN}_{2} \mathrm{O}_{2}$ : C, 67.87; H, 8.11; N, 5.46. Found C, 67.94; H, 8.11; N, 5.52 


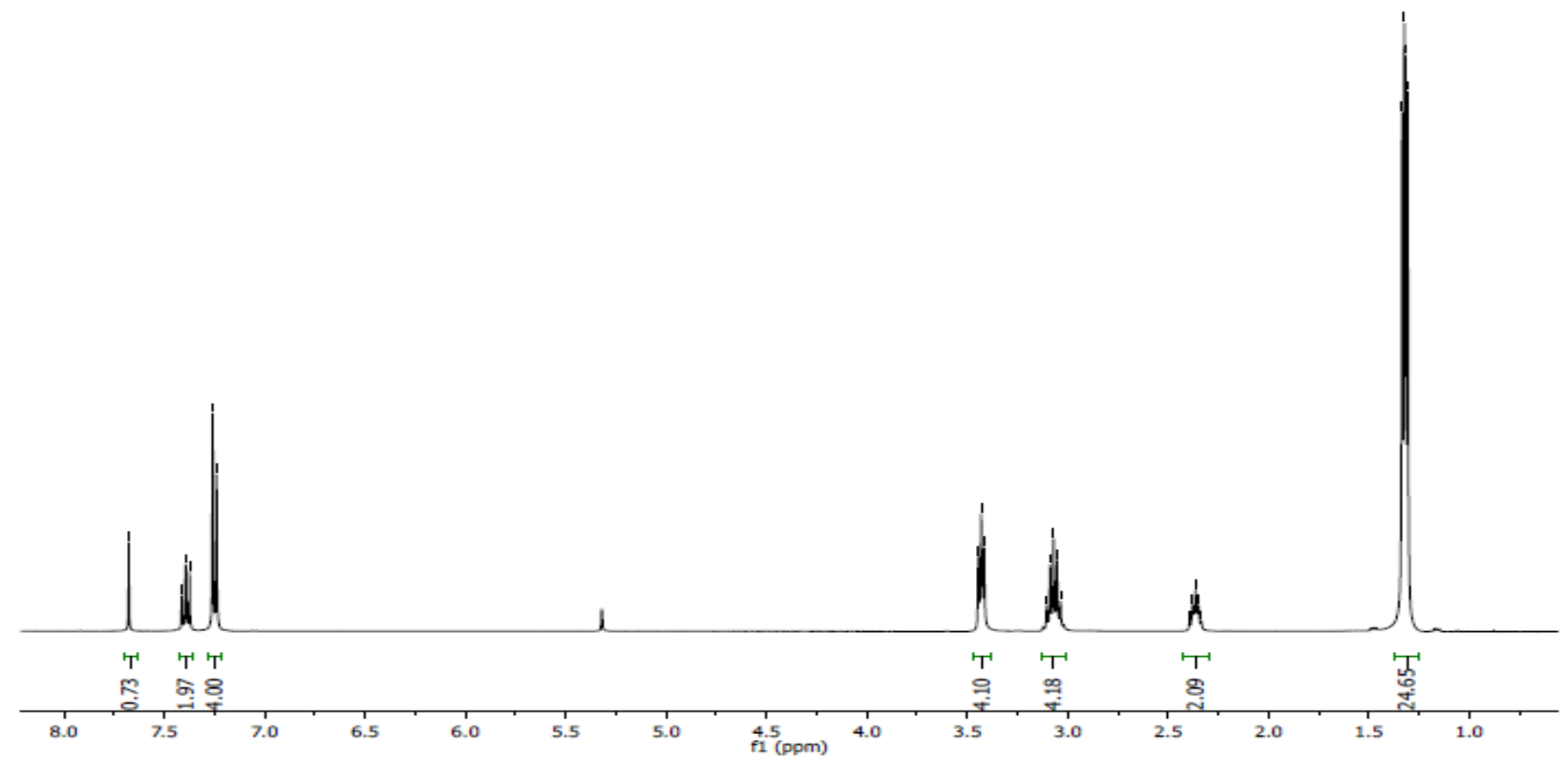

Figure S8. ${ }^{1} \mathrm{H}$ NMR spectrum of (6Dipp)copper(I) formate in $\mathrm{CD}_{2} \mathrm{Cl}_{2}$.

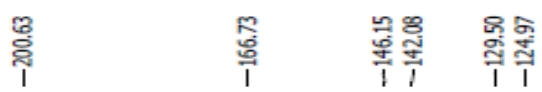
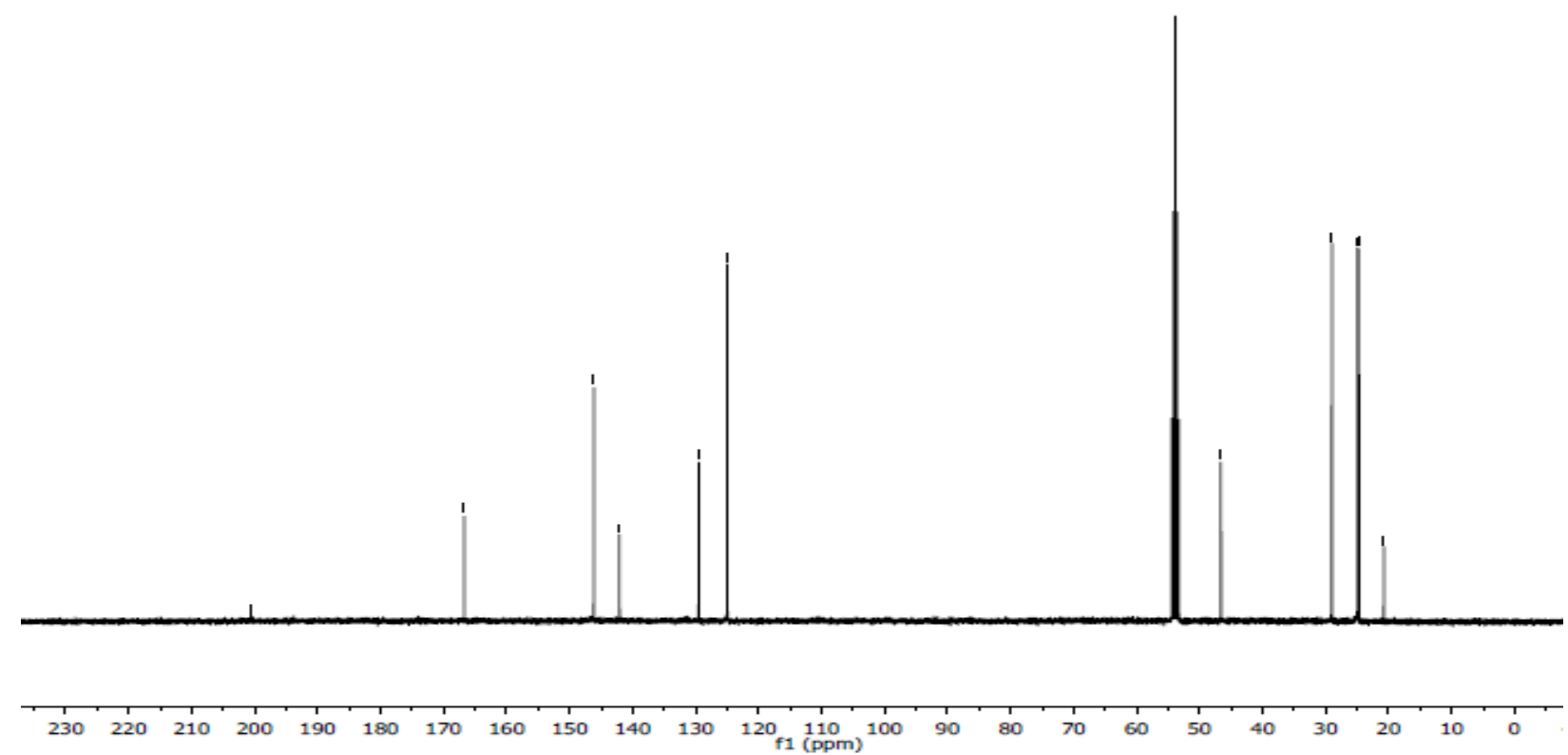

Figure S9. ${ }^{13} \mathrm{C}$ NMR spectrum of (6Dipp)copper(I) formate in $\mathrm{CD}_{2} \mathrm{Cl}_{2}$. 


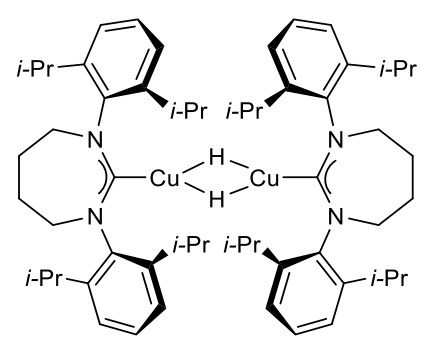

[(7Dipp)CuH $]_{2}$ (3b). Pinacolborane 4,4,5,5,- tetramethyl-1,3,2-dioxaborolane [HB(pin)] (0.040 mL, $0.26 \mathrm{mmol})$ was added to a solution of (7Dipp) $\mathrm{Cu}(\mathrm{O} t-\mathrm{Bu})(0.102 \mathrm{~g}, 0.184 \mathrm{mmol})$ in THF $(2 \mathrm{~mL})$ via syringe. The resulting yellow solution was layered with pentane $(2 \mathrm{~mL})$ and placed in the freezer at $-35^{\circ} \mathrm{C}$ for $12 \mathrm{~h}$. The supernatant was decanted by pipette before pentane $(2 \mathrm{~mL})$ was added and placed back in the freezer for $4 \mathrm{~h}$. The supernatant was decanted and pentane $(2 \mathrm{~mL})$ was added again before being placed in the freezer for another $4 \mathrm{~h}$. The resulting orange crystals were dried in vacuo for $1 \mathrm{~h}$ to afford the title complex. $(0.080 \mathrm{~g}, 90 \%){ }^{1} \mathrm{H}$ NMR $\left(400 \mathrm{MHz}, \mathrm{C}_{6} \mathrm{D}_{6}\right)$ : $\delta(\mathrm{ppm}) 7.20$ $\left(\mathrm{t},{ }^{3} J_{\mathrm{HH}}=7.6 \mathrm{~Hz}, 2 \mathrm{H}\right.$, para-CH$), 6.99\left(\mathrm{~d},{ }^{3} J_{\mathrm{HH}}=7.6 \mathrm{~Hz}, 4 \mathrm{H}\right.$, meta-CH$), 3.41\left(\mathrm{~m}, 4 \mathrm{H}, \mathrm{NCH}_{2}\right), 3.31$ (sept, $\left.{ }^{3} J_{\mathrm{HH}}=6.8 \mathrm{~Hz}, 4 \mathrm{H}, \mathrm{CH}\left(\mathrm{CH}_{3}\right)_{2}\right), 1.74\left(\mathrm{~m}, 4 \mathrm{H}, \mathrm{NCH}_{2} \mathrm{CH}_{2}\right), 1.41\left(\mathrm{~d},{ }^{3} J_{\mathrm{HH}}=6.8 \mathrm{~Hz}, 12 \mathrm{H}\right.$, $\left.\mathrm{CH}_{2}\left(\mathrm{CH}_{3}\right)_{2}\right), 1.30\left(\mathrm{~d},{ }^{3} \mathrm{~J}_{\mathrm{HH}}=6.8 \mathrm{~Hz}, 12 \mathrm{H}, \mathrm{CH}_{2}\left(\mathrm{CH}_{3}\right)_{2}\right), 0.47(\mathrm{~s}, 1 \mathrm{H}, \mathrm{Cu}-\mathrm{H}) \cdot{ }^{13} \mathrm{C}\left\{{ }^{1} \mathrm{H}\right\} \mathrm{NMR}(100$ MHz, $\mathrm{C}_{6} \mathrm{D}_{6}$ ): $\delta(\mathrm{ppm}) 223.9$ (NCCu), 145.4 (ortho-C), 144.8 (ipso-C), 127.7 (meta-C), 124.7 (paraC), $53.7\left(\mathrm{NCH}_{2}\right), 28.8\left(\mathrm{CH}\left(\mathrm{CH}_{3}\right)_{2}\right), 26.0\left(\mathrm{NCH}_{2} \mathrm{CH}_{2}\right), 25.7\left(\mathrm{CH}\left(\mathrm{CH}_{3}\right)_{2}\right), 25.6\left(\mathrm{CH}\left(\mathrm{CH}_{3}\right)_{2}\right), \mathrm{IR}: v$ $\left(\mathrm{cm}^{-1}\right) 2956,2861,1459,1420,1382,1360,1317,1267,1176,1057,936,912,798,782,752,618$, $550,454,401$.

Note: We have been unable to obtain satisfactory elemental analysis for $\mathbf{3 b}$. The complex is extremely air and moisture-sensitive. After exposure of a benzene solution of $\mathbf{3 b}$ to dry $\mathrm{CO}_{2}$, and concentration in vacuo, the more stable formate complex $\mathbf{4 b}$ was isolated without further purification and its elemental analysis was obtained. (See p. S-16) We reasoned that only a sufficiently pure sample of $\mathbf{4 b}$ would give rise to analytically pure $\mathbf{4 b}$. 


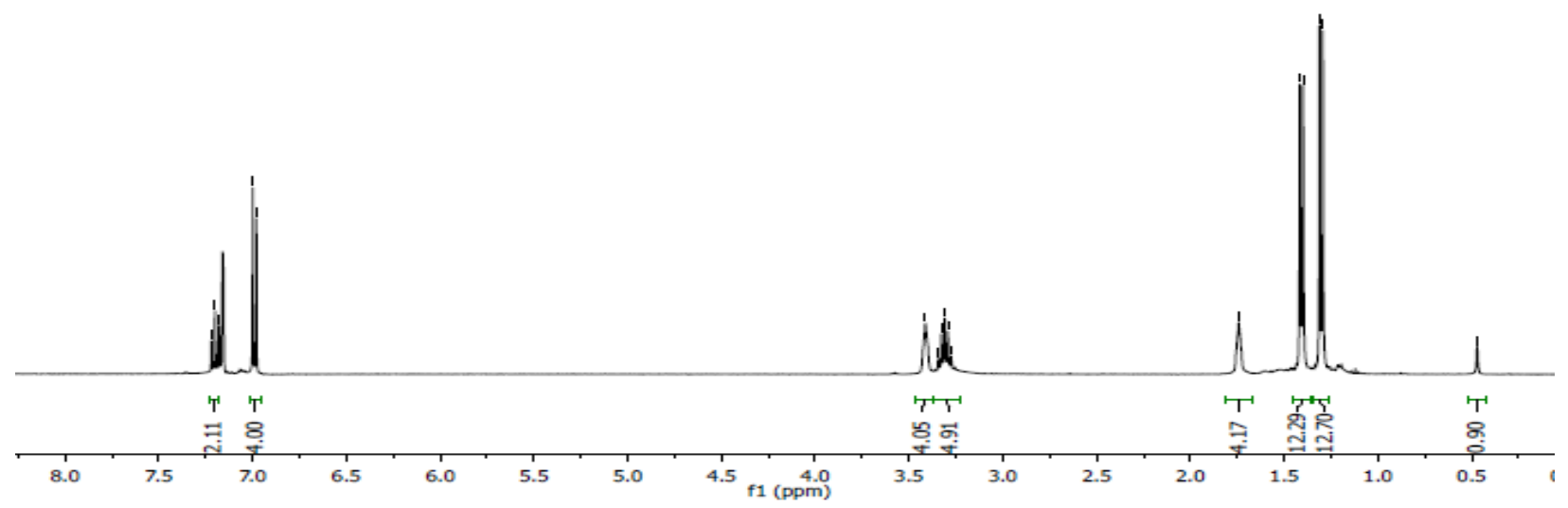

Figure S10. ${ }^{1} \mathrm{H}$ NMR spectrum of $[(7 \mathrm{Dipp}) \mathrm{CuH}]_{2}$ in $\mathrm{C}_{6} \mathrm{D}_{6}$.
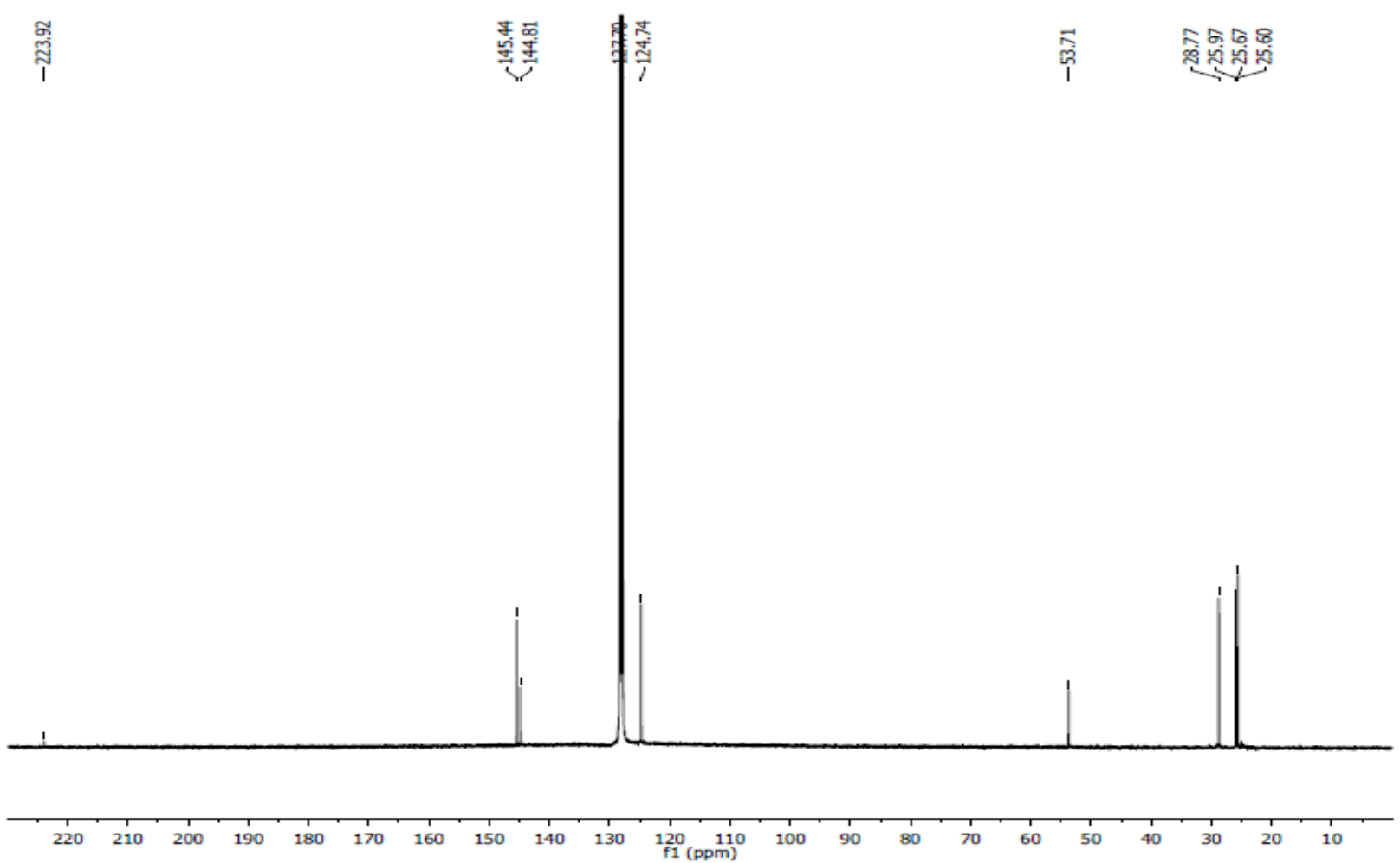

Figure S11. ${ }^{13} \mathrm{C}$ NMR spectrum of $[(7 \mathrm{Dipp}) \mathrm{CuH}]_{2}$ in $\mathrm{C}_{6} \mathrm{D}_{6}$. 


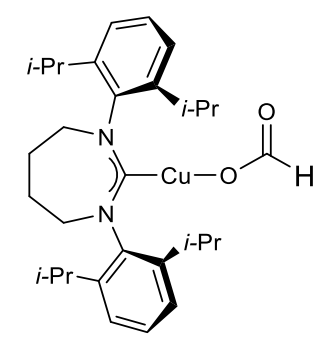

(7Dipp)copper(I) formate (4b) In an oven dried $25 \mathrm{~mL}$ Schlenk flask under $\mathrm{N}_{2}$, a solution of [(7Dipp $) \mathrm{CuH}]_{2}(0.022 \mathrm{~g}, 0.023 \mathrm{mmol})$ in benzene $(5 \mathrm{~mL})$ was degassed by 3 freeze-pump-thaw cycles before being exposed to an atmosphere of $\mathrm{CO}_{2}$. The bright orange solution immediately became colorless, and deposited a white precipitate. The benzene was removed in vacuo and dried under vacuum for $12 \mathrm{~h}$ affording the product as a colorless powder. $(0.021 \mathrm{~g}, 85 \%){ }^{1} \mathrm{H}$ NMR $(400$ $\left.\mathrm{MHz}, \mathrm{CD}_{2} \mathrm{Cl}_{2}\right): \delta(\mathrm{ppm}) 7.59(\mathrm{~s}, 1 \mathrm{H}, \mathrm{OC}(\mathrm{O}) H) 7.35\left(\mathrm{t},{ }^{3} J_{\mathrm{HH}}=7.6 \mathrm{~Hz}, 2 \mathrm{H}\right.$, para-CH$), 7.23\left(\mathrm{~d},{ }^{3} J_{\mathrm{HH}}=\right.$ $7.6 \mathrm{~Hz}, 4 \mathrm{H}$, meta-CH), $3.97\left(\mathrm{~m}, 4 \mathrm{H}, \mathrm{NCH}_{2}\right), 3.26\left(\mathrm{sept},{ }^{3} J_{\mathrm{HH}}=6.8 \mathrm{~Hz}, 4 \mathrm{H}, \mathrm{CH}\left(\mathrm{CH}_{3}\right)_{2}\right), 2.33(\mathrm{~m}, 4 \mathrm{H}$, $\left.\mathrm{NCH}_{2} \mathrm{CH}_{2}\right), 1.33\left(\mathrm{~d},{ }^{3} J_{\mathrm{HH}}=7.2 \mathrm{~Hz}, 24 \mathrm{H}, \mathrm{CH}_{2}\left(\mathrm{CH}_{3}\right)_{2}\right) .{ }^{13} \mathrm{C}\left\{{ }^{1} \mathrm{H}\right\} \mathrm{NMR}\left(100 \mathrm{MHz}, \mathrm{CD}_{2} \mathrm{Cl}_{2}\right): \delta(\mathrm{ppm})$ $210.1(\mathrm{NCCu}), 166.7(\mathrm{OC}(\mathrm{O}) \mathrm{H}) 145.5$ (ortho-C), 144.5 (ipso-C), 129.1 (meta-C), 125.1 (para-C), $54.4\left(\mathrm{NCH}_{2}\right), 37.0\left(\mathrm{OC}\left(\mathrm{CH}_{3}\right)_{3}\right), 29.1\left(\mathrm{CH}\left(\mathrm{CH}_{3}\right)_{2}\right), 25.1\left(\mathrm{NCH}_{2} \mathrm{CH}_{2}\right), 24.74\left(\mathrm{CH}\left(\mathrm{CH}_{3}\right)_{2}\right), 24.70$ $\left(\mathrm{CH}\left(\mathrm{CH}_{3}\right)_{2}\right) . \mathrm{IR}: v\left(\mathrm{~cm}^{-1}\right)$ 2962, 2926, 2866, 2795, 1634, 1590, 1478, 1468, 1446, 1387, 1364, 1316, 1290, 1266, 1179, 1095, 1057, 949, 936, 805, 785, 762, 695, 496, 452. Anal. Calcd for $\mathrm{C}_{30} \mathrm{H}_{43} \mathrm{CuN}_{2} \mathrm{O}_{2}$ : C, 68.34; H, 8.22; N, 5.31. Found C, 68.10; H, 8.37; N, 5.18. 


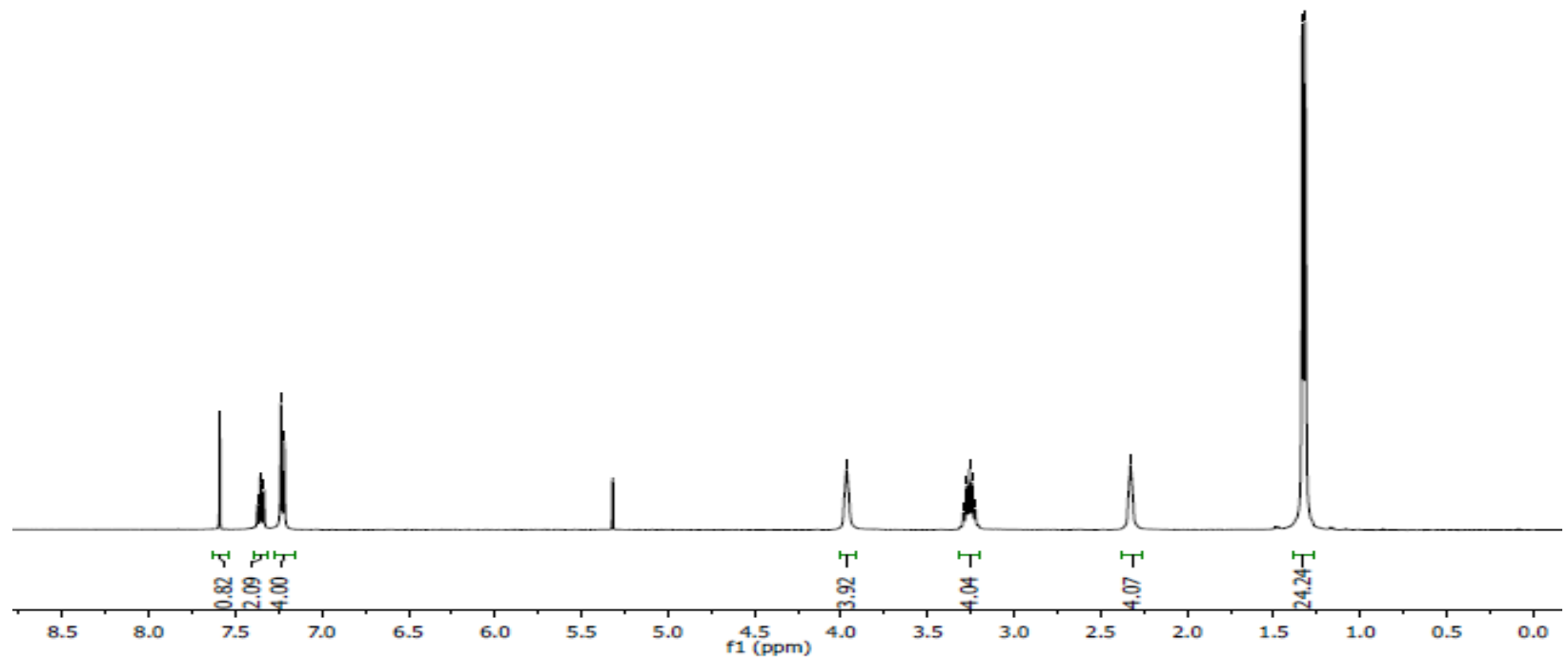

Figure S12. ${ }^{1} \mathrm{H}$ NMR spectrum of (7Dipp)copper(I) formate in $\mathrm{CD}_{2} \mathrm{Cl}_{2}$.

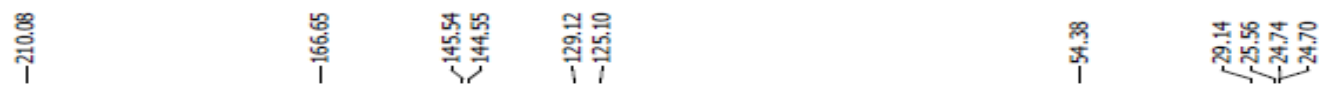

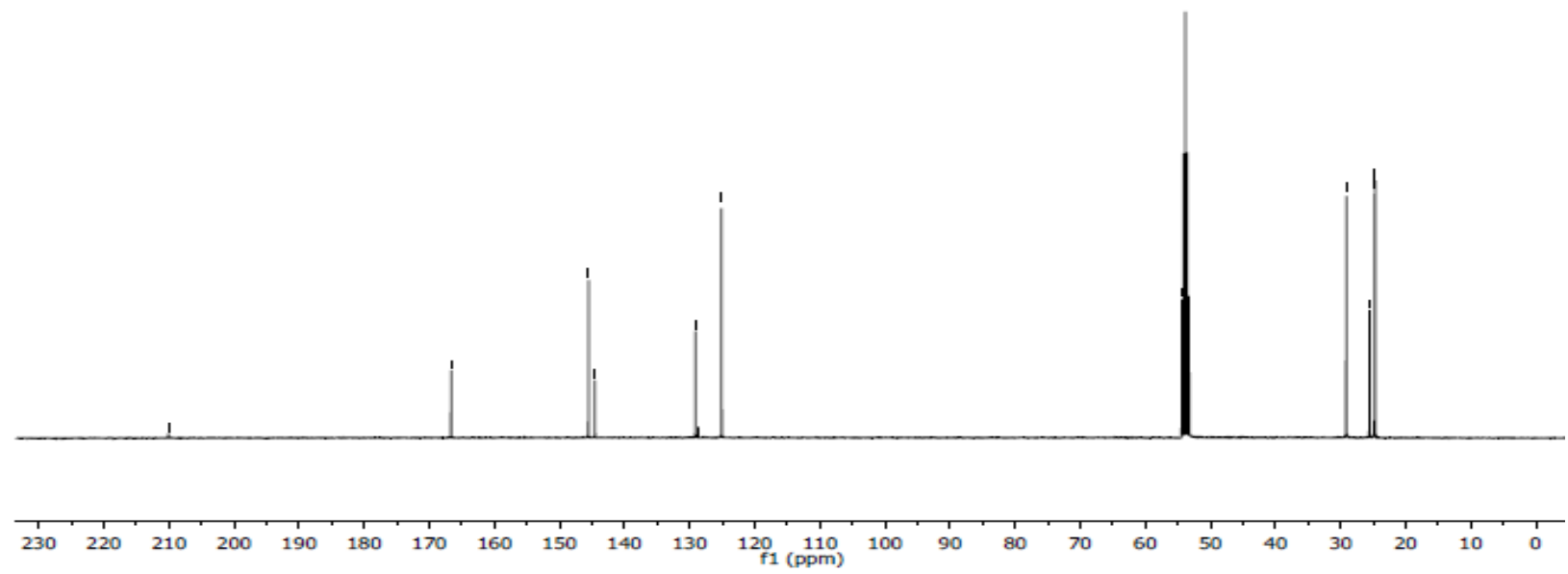

Figure S13. ${ }^{13} \mathrm{C}$ NMR spectrum of (7Dipp)copper(I) formate in $\mathrm{CD}_{2} \mathrm{Cl}_{2}$. 


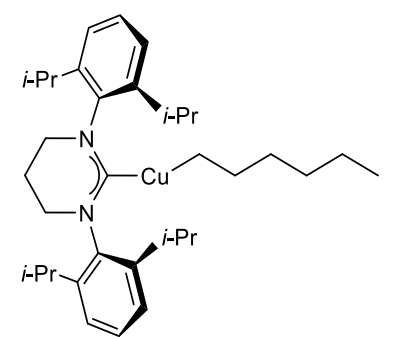

(6Dipp)copper(I) hexyl (5a). 1-Hexene $(0.106 \mathrm{~mL}, 0.85 \mathrm{mmol})$ was added to a solution of $[(6 \mathrm{Dipp}) \mathrm{CuH}]_{2}(0.020 \mathrm{~g}, 0.021 \mathrm{mmol})$ in THF $(2 \mathrm{~mL})$. After $24 \mathrm{~h}$ the resulting solution was concentrated in vacuo to give a colorless solid $(0.017 \mathrm{~g}, 72 \%) .{ }^{1} \mathrm{H}$ NMR $\left(400 \mathrm{MHz}, \mathrm{C}_{6} \mathrm{D}_{6}\right): \delta(\mathrm{ppm})$ $7.21\left(\mathrm{dd},{ }^{3} J_{\mathrm{HH}}=8.4,7.2 \mathrm{~Hz}, 2 \mathrm{H}\right.$, para- $\left.\mathrm{CH}\right), 7.10\left(\mathrm{~d},{ }^{3} J_{\mathrm{HH}}=7.6 \mathrm{~Hz}, 4 \mathrm{H}\right.$, meta- $\left.\mathrm{CH}\right), 3.08\left(\mathrm{sept},{ }^{3} J_{\mathrm{HH}}=\right.$ $\left.6.8 \mathrm{~Hz}, 4 \mathrm{H}, \mathrm{CH}\left(\mathrm{CH}_{3}\right)_{2}\right), 2.70\left(\mathrm{t},{ }^{3} J_{\mathrm{HH}}=6.0 \mathrm{~Hz}, 4 \mathrm{H}, \mathrm{NCH} 2\right), 1.61\left(\right.$ quin, ${ }^{3} J_{\mathrm{HH}}=7.6 \mathrm{~Hz}, 2 \mathrm{H}, \mathrm{Cu}-$ $\left.\mathrm{CH}_{2} \mathrm{CH}_{2}\right), 1.54\left(\mathrm{~d},{ }^{3} J_{\mathrm{HH}}=6.8 \mathrm{~Hz}, 12 \mathrm{H}, \mathrm{CH}_{2}\left(\mathrm{CH}_{3}\right)_{2}\right), 1.50\left(\mathrm{~m}, 2 \mathrm{H}, \mathrm{NCH}_{2} \mathrm{CH}_{2}\right), 1.36(\mathrm{~m}, 4 \mathrm{H}, \mathrm{Cu}-$ $\left.\left(\mathrm{CH}_{2}\right)_{2}\left(\mathrm{CH}_{2}\right)_{2}\right), 1.22\left(\mathrm{~d},{ }^{3} \mathrm{~J}_{\mathrm{HH}}=6.8 \mathrm{~Hz}, 12 \mathrm{H}, \mathrm{CH}_{2}\left(\mathrm{CH}_{3}\right)_{2}\right), 1.09$ (quin, ${ }^{3} J_{\mathrm{HH}}=7.6 \mathrm{~Hz}, 2 \mathrm{H}, \mathrm{Cu}-$ $\left.\left(\mathrm{CH}_{2}\right)_{4}\left(\mathrm{CH}_{2}\right) \mathrm{CH}_{3}\right), 0.97\left(\mathrm{t},{ }^{3} J_{\mathrm{HH}}=6.8 \mathrm{~Hz}, 3 \mathrm{H}, \mathrm{Cu}-\left(\mathrm{CH}_{2}\right)_{5} \mathrm{CH}_{3}\right), 0.12\left(\mathrm{t},{ }^{3} J_{\mathrm{HH}}=7.6 \mathrm{~Hz}, 2 \mathrm{H}, \mathrm{Cu}-\mathrm{CH}_{2}\right)$ ${ }^{13} \mathrm{C}\left\{{ }^{1} \mathrm{H}\right\}$ NMR (100 MHz, $\left.\mathrm{C}_{6} \mathrm{D}_{6}\right): \delta(\mathrm{ppm}) 204.7(\mathrm{NCCu}), 145.8$ (ortho-C), 142.1 (ipso-C), 129.0 (meta-C), 124.5 (para-C), $46.0\left(\mathrm{NCH}_{2}\right), 38.0\left(\mathrm{Cu}-\mathrm{CH}_{2} \mathrm{CH}_{2}\right), 33.1\left(\mathrm{Cu}-\left(\mathrm{CH}_{2}\right)_{2} \mathrm{CH}_{2}\right), 31.1(\mathrm{Cu}-$ $\left.\left(\mathrm{CH}_{2}\right)_{3} \mathrm{CH}_{2}\right), 28.9\left(\mathrm{CH}\left(\mathrm{CH}_{3}\right)_{2}\right), 25.0\left(\mathrm{CH}\left(\mathrm{CH}_{3}\right)_{2}\right), 24.7\left(\mathrm{CH}\left(\mathrm{CH}_{3}\right)_{2}\right), 23.6\left(\mathrm{Cu}-\left(\mathrm{CH}_{2}\right)_{4} \mathrm{CH}_{2}\right), 20.5$ $\left(\mathrm{NCH}_{2} \mathrm{CH}_{2}\right), 14.9\left(\mathrm{Cu}-\left(\mathrm{CH}_{2}\right)_{5} \mathrm{CH}_{3}\right), 10.8\left(\mathrm{Cu}-\mathrm{CH}_{2}\right) . \mathrm{IR} v\left(\mathrm{~cm}^{-1}\right)$ 2960, 2867, 1645, 1628, 1506, 1452, $1303,1203,1056,935,803,757,556,537,492,452,420,401$

Note: We have been unable to obtain satisfactory elemental analysis for $\mathbf{5 a}$. The complex is extremely air, moisture and light sensitive. While NMR-silent impurities cannot be ruled out, we believe the ${ }^{1} \mathrm{H}$ and ${ }^{13} \mathrm{C}$ NMR spectra provided reflect the purity of the sample. 


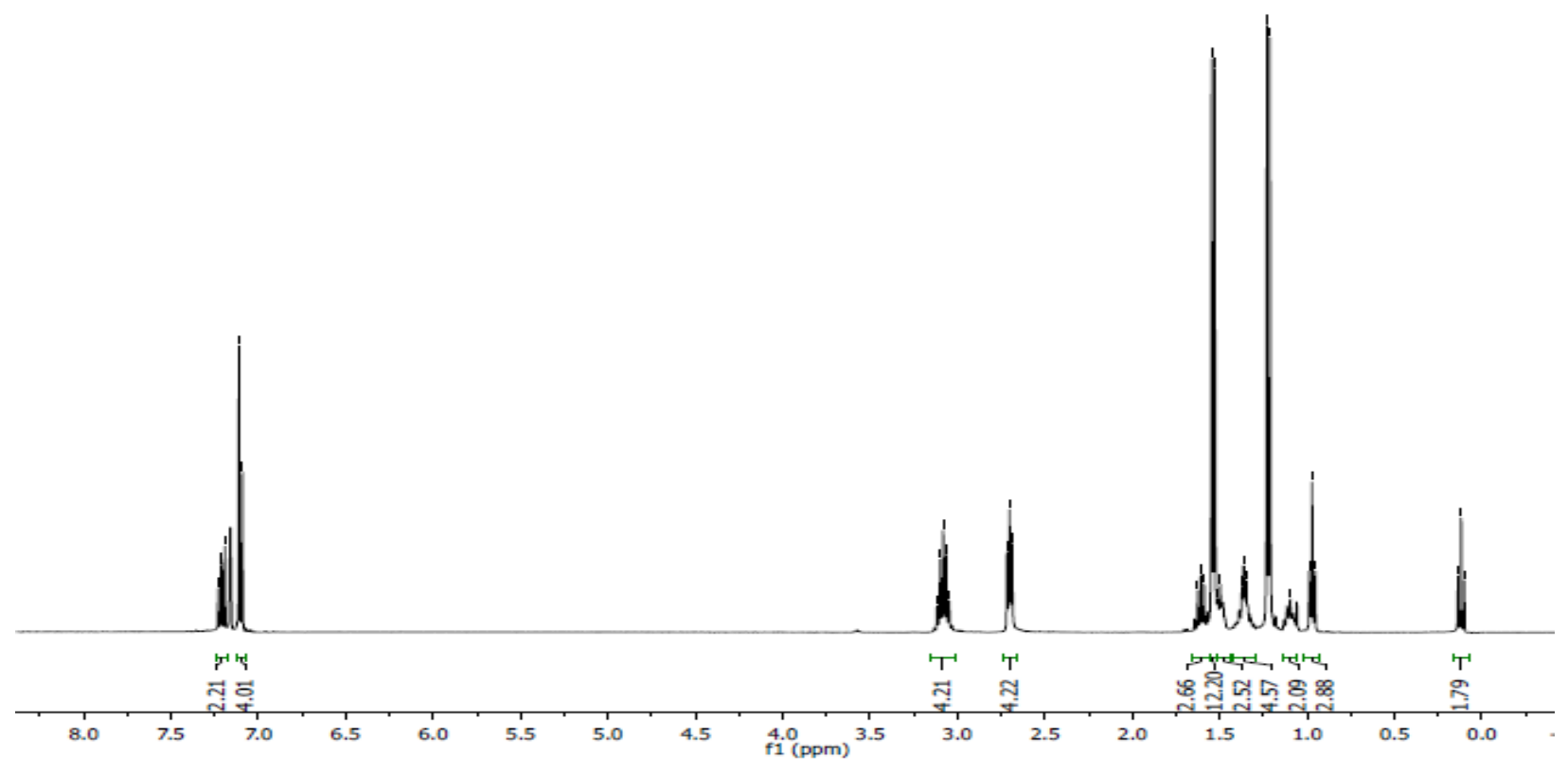

Figure S14. ${ }^{1} \mathrm{H}$ NMR spectrum of (6Dipp)copper(I) hexyl in $\mathrm{C}_{6} \mathrm{D}_{6}$.

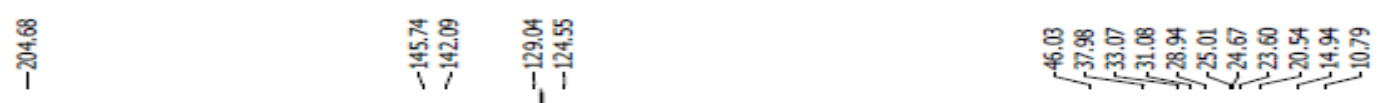

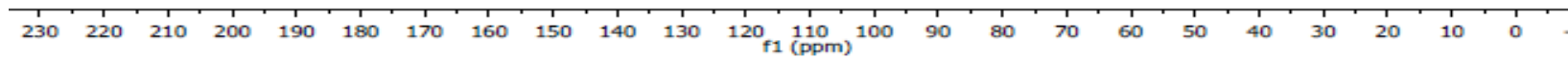

Figure S15. ${ }^{13} \mathrm{C}$ NMR spectrum of (6Dipp)copper(I) hexyl in $\mathrm{C}_{6} \mathrm{D}_{6}$. 


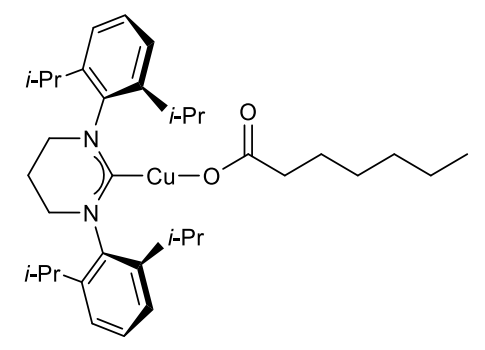

(6Dipp)copper(I) heptanoate (6a). In an oven-dried J. Young NMR tube under $\mathrm{N}_{2}$, a solution of (6Dipp)Cu-hexyl $(0.030 \mathrm{~g}, 0.054 \mathrm{mmol})$ in $\mathrm{C}_{6} \mathrm{D}_{6}(0.7 \mathrm{~mL})$ was degassed by 3 freeze-pump-thaw cycles before being exposed to an atmosphere of $\mathrm{CO}_{2}$. After $40 \mathrm{~min}$, the $\mathrm{C}_{6} \mathrm{D}_{6}$ was removed in vacuo and the residual solid was dried for $12 \mathrm{~h}$, affording the product as a colorless powder. $(0.029 \mathrm{~g}$, 91\%). ${ }^{1} \mathrm{H}$ NMR (400 MHz, $\left.\mathrm{C}_{6} \mathrm{D}_{6}\right): \delta(\mathrm{ppm}) 7.16\left(\mathrm{t},{ }^{3} J_{\mathrm{HH}}=7.2 \mathrm{~Hz}, 2 \mathrm{H}\right.$, para-CH), $7.07\left(\mathrm{~d},{ }^{3} J_{\mathrm{HH}}=7.2\right.$ $\mathrm{Hz}, 4 \mathrm{H}$, meta-CH), $3.04\left(\mathrm{sept},{ }^{3} J_{\mathrm{HH}}=6.8 \mathrm{~Hz}, 4 \mathrm{H}, \mathrm{CH}\left(\mathrm{CH}_{3}\right)_{2}\right), 2.72\left(\mathrm{t},{ }^{3} J_{\mathrm{HH}}=6.0 \mathrm{~Hz}, 4 \mathrm{H}, \mathrm{NCH}\right)_{2}$, $2.12\left(\mathrm{t},{ }^{3} J_{\mathrm{HH}}=7.2 \mathrm{~Hz}, 2 \mathrm{H}, \mathrm{OC}(\mathrm{O}) \mathrm{CH}_{2}\right), 1.57\left(\mathrm{~d},{ }^{3} J_{\mathrm{HH}}=7.2 \mathrm{~Hz}, 12 \mathrm{H}, \mathrm{CH}_{2}\left(\mathrm{CH}_{3}\right)_{2}\right), 1.54(\mathrm{~m}, 4 \mathrm{H}$, $\left.\mathrm{OC}(\mathrm{O}) \mathrm{CH}_{2} \mathrm{CH}_{2}, \mathrm{NCH}_{2} \mathrm{CH}_{2}\right), 1.18\left(\mathrm{~d},{ }^{3} \mathrm{~J}_{\mathrm{HH}}=7.2 \mathrm{~Hz}, 12 \mathrm{H}, \mathrm{CH}_{2}\left(\mathrm{CH}_{3}\right)_{2}\right), 1.11(\mathrm{~m}, 6 \mathrm{H}, \mathrm{OC}(\mathrm{O})$ $\left.\left(\mathrm{CH}_{2}\right)_{2}\left(\mathrm{CH}_{2}\right)_{3}\right), 0.80\left(\mathrm{t},{ }^{3} J_{\mathrm{HH}}=7.2 \mathrm{~Hz}, 3 \mathrm{H}, \mathrm{OC}(\mathrm{O})\left(\mathrm{CH}_{2}\right)_{5} \mathrm{CH}_{3}\right) \cdot{ }^{13} \mathrm{C}\left\{{ }^{1} \mathrm{H}\right\} \mathrm{NMR}\left(100 \mathrm{MHz}, \mathrm{C}_{6} \mathrm{D}_{6}\right): \delta$ (ppm) $202.1(\mathrm{NCCu}), 178.8(\mathrm{Cu}-\mathrm{OC}(\mathrm{O}), 145.7$ (ortho-C), 142.1 (ipso-C), 129.5 (meta-C), 124.9 (para-C), $46.0\left(\mathrm{NCH}_{2}\right), 37.0\left(\mathrm{OC}(\mathrm{O}) \mathrm{CH}_{2} \mathrm{CH}_{2}\right), 32.2\left(\mathrm{OC}(\mathrm{O}) \mathrm{CH}_{2} \mathrm{CH}_{2}\right), 29.6\left(\mathrm{OC}(\mathrm{O})\left(\mathrm{CH}_{2}\right)_{2} \mathrm{CH}_{2}\right)$, $28.9\left(\mathrm{CH}\left(\mathrm{CH}_{3}\right)_{2}\right), 26.9\left(\mathrm{OC}(\mathrm{O})\left(\mathrm{CH}_{2}\right)_{3} \mathrm{CH}_{2}\right), 25.1\left(\mathrm{CH}\left(\mathrm{CH}_{3}\right)_{2}\right), 24.8\left(\mathrm{CH}\left(\mathrm{CH}_{3}\right)_{2}\right), 23.0$ $\left(\mathrm{OC}(\mathrm{O})\left(\mathrm{CH}_{2}\right)_{4} \mathrm{CH}_{2}\right), 14.4\left(\mathrm{OC}(\mathrm{O})\left(\mathrm{CH}_{2}\right)_{5} \mathrm{CH}_{3}\right) . \mathrm{IR} v\left(\mathrm{~cm}^{-1}\right)$ 2959, 2925, 2866, 1607, 1509, 1554, $1371,1342,1306,1235,1205,1152,1057,984,935,806,762,726,638,556,502,453$.

Note: We have been unable to obtain satisfactory elemental analysis for 6a. The complex is air- and moisture-sensitive. While NMR silent impurities cannot be ruled out, we believe the ${ }^{1} \mathrm{H}$ and ${ }^{13} \mathrm{C}$ NMR spectra reflect the purity of the sample. 


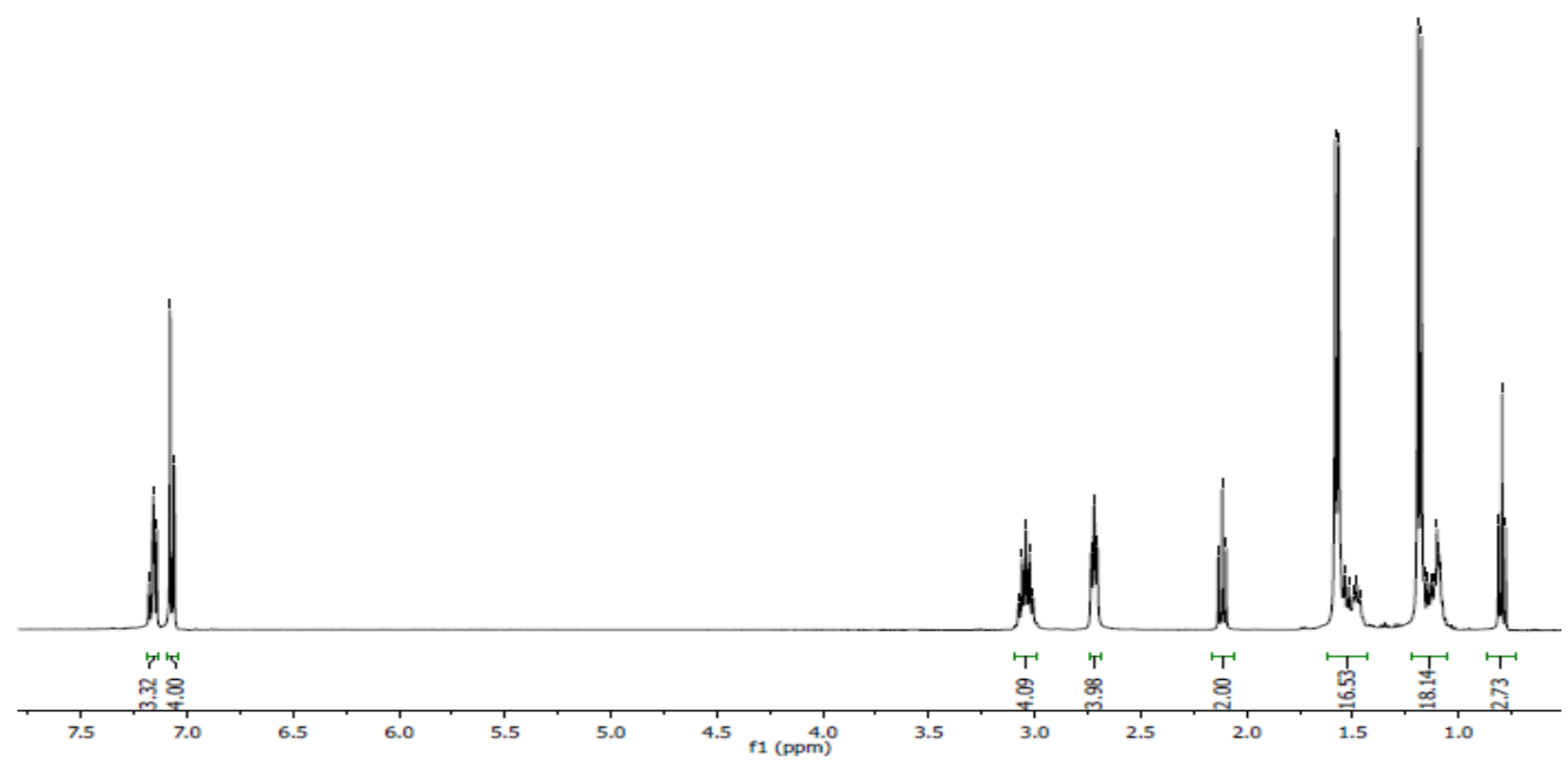

Figure S16. ${ }^{1}$ H NMR spectrum of (6Dipp)copper(I) heptanoate in $\mathrm{C}_{6} \mathrm{D}_{6}$.
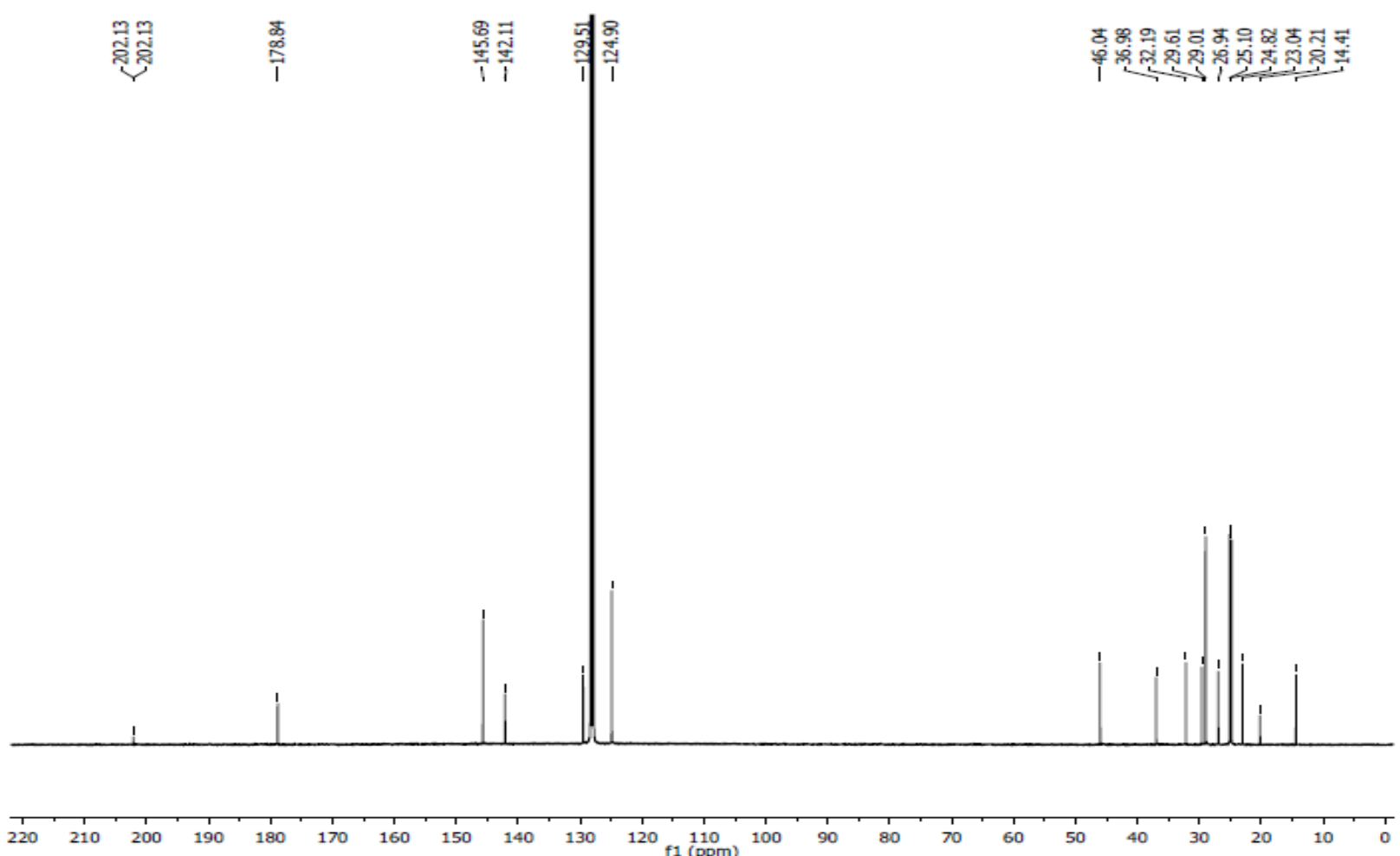

Figure S17. ${ }^{13} \mathrm{C}$ NMR spectrum of (6Dipp)copper(I) heptanoate in $\mathrm{C}_{6} \mathrm{D}_{6}$. 


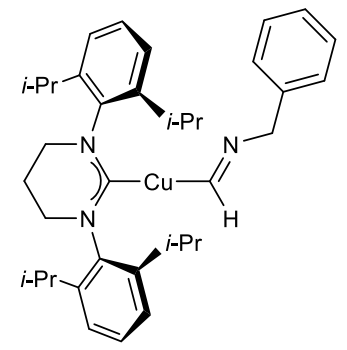

(6Dipp)copper(I) ( $N$-benzyl)formimidoyl (7a). Benzyl isocyanide (0.064 mL, $0.052 \mathrm{mmol})$ was added via syringe to a solution of $[(6 \mathrm{Dipp}) \mathrm{CuH}]_{2}(0.025 \mathrm{~g}, 0.026 \mathrm{mmol})$ toluene $(1.5 \mathrm{~mL})$. After $3 \mathrm{~h}$ the solution was layered with pentane $(1 \mathrm{~mL})$ and allowed to stand in the freezer at $-35^{\circ} \mathrm{C}$ for $48 \mathrm{~h}$. The supernatant was decanted by pipette, the resulting crystals were redissolved in toluene $(1 \mathrm{~mL})$, layered with pentane $(1 \mathrm{~mL})$ and placed back in the freezer. After $48 \mathrm{~h}$, the supernatant was decanted by pipette. The resulting crystals were dried in vacuo for $12 \mathrm{~h}$ to give a pale yellow solid. $(0.018 \mathrm{~g}, 57 \%){ }^{1} \mathrm{H}$ NMR $\left(400 \mathrm{MHz}, \mathrm{C}_{6} \mathrm{D}_{6}\right): \delta(\mathrm{ppm}) 9.37(\mathrm{~s}, 1 \mathrm{H}, \mathrm{Cu}-\mathrm{C}(\mathrm{N}) H), 7.26\left(\mathrm{~m}, 5 \mathrm{H},-\mathrm{C}_{6} H_{5}\right)$, $7.18\left(\mathrm{t},{ }^{3} J_{\mathrm{HH}}=7.2 \mathrm{~Hz}\right.$, para-CH), $7.08\left(\mathrm{~d},{ }^{3} \mathrm{~J}_{\mathrm{HH}}=7.2 \mathrm{~Hz}, 4 \mathrm{H}\right.$, meta-CH), $4.53\left(\mathrm{~s}, 2 \mathrm{H}, \mathrm{C}(\mathrm{H})=\mathrm{NCH}{ }_{2}\right)$, $3.06\left(\mathrm{sept},{ }^{3} J_{\mathrm{HH}}=6.8 \mathrm{~Hz}, 4 \mathrm{H}, \mathrm{CH}\left(\mathrm{CH}_{3}\right)_{2}\right), 2.70\left(\mathrm{t},{ }^{3} J_{\mathrm{HH}}=6.0 \mathrm{~Hz}, 4 \mathrm{H}, \mathrm{NCH}\right), 1.56\left(\mathrm{~d},{ }^{3} J_{\mathrm{HH}}=6.8 \mathrm{~Hz}\right.$, $\left.12 \mathrm{H}, \mathrm{CH}_{2}\left(\mathrm{CH}_{3}\right)_{2}\right), 1.47$ (quin, $\left.{ }^{3} J_{\mathrm{HH}}=5.6 \mathrm{~Hz}, 2 \mathrm{H}, \mathrm{NCH}_{2} \mathrm{CH}_{2}\right), 1.20\left(\mathrm{~d},{ }^{3} J_{\mathrm{HH}}=7.2 \mathrm{~Hz}, 12 \mathrm{H}\right.$, $\left.\mathrm{CH}_{2}\left(\mathrm{CH}_{3}\right)_{2}\right) .{ }^{13} \mathrm{C}\left\{{ }^{1} \mathrm{H}\right\} \mathrm{NMR}\left(100 \mathrm{MHz}, \mathrm{C}_{6} \mathrm{D}_{6}\right): \delta(\mathrm{ppm}) 209.9(\mathrm{Cu}-\mathrm{C}(\mathrm{N}) \mathrm{H}), 204.8(\mathrm{NCCu}), 145.8$ (dipp-ortho-C), 144.1 (Bn-ipso-C), 141.7 (dipp-ipso-C), 129.3 (dipp-meta-C), 128.2 (Bn-ortho-C), 127.9 (Bn-meta-C), 125.4 (Bn-para-C), 124.7 (dipp-para-C), $77.1\left(\mathrm{C}(\mathrm{H})=\mathrm{NCH}_{2}\right), 45.9\left(\mathrm{NCH}_{2}\right), 29.0$ $\left(\mathrm{CH}\left(\mathrm{CH}_{3}\right)_{2}\right), 25.2\left(\mathrm{CH}\left(\mathrm{CH}_{3}\right)_{2}\right), 24.7\left(\mathrm{CH}\left(\mathrm{CH}_{3}\right)_{2}\right), 20.4\left(\mathrm{NCH}_{2} \mathrm{CH}_{2}\right) . \mathrm{IR} v\left(\mathrm{~cm}^{-1}\right) 2959,2923,2866$, $1582,1509,1451,1400,1384,1254,1204,1056,989,934,8789,804,754,694,618,551,512,452$

Note: We have been unable to obtain satisfactory elemental analysis for $7 \mathbf{a}$. The complex is extremely air-, and moisture-sensitive. Although NMR silent impurities cannot be ruled out, we believe the ${ }^{1} \mathrm{H}$ and ${ }^{13} \mathrm{C}$ NMR spectra reflect the purity of the sample. 

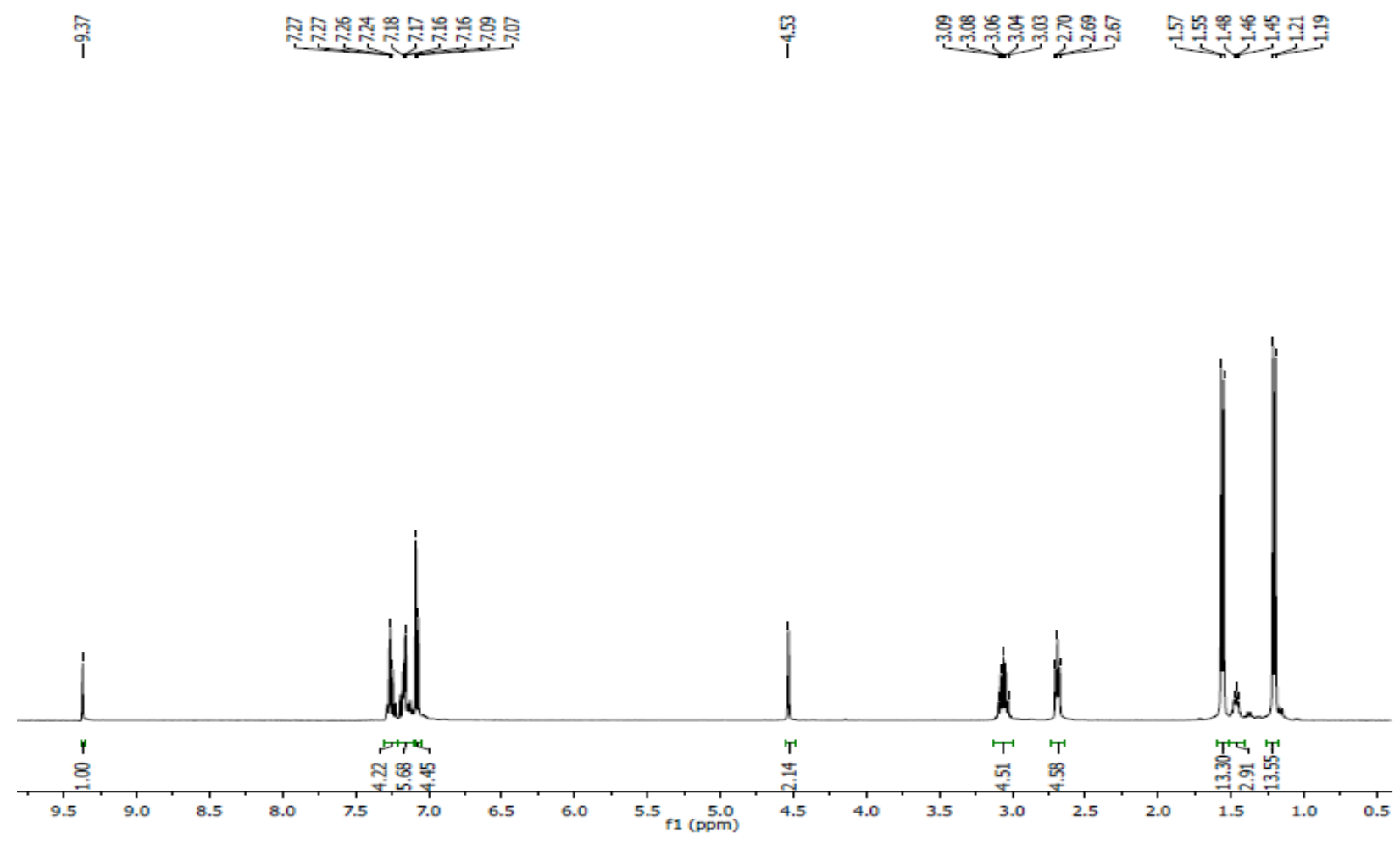

Figure S18. ${ }^{1} \mathrm{H}$ NMR spectrum of (6Dipp)copper(I) ( $N$-benzyl)formimidoyl in $\mathrm{C}_{6} \mathrm{D}_{6}$.

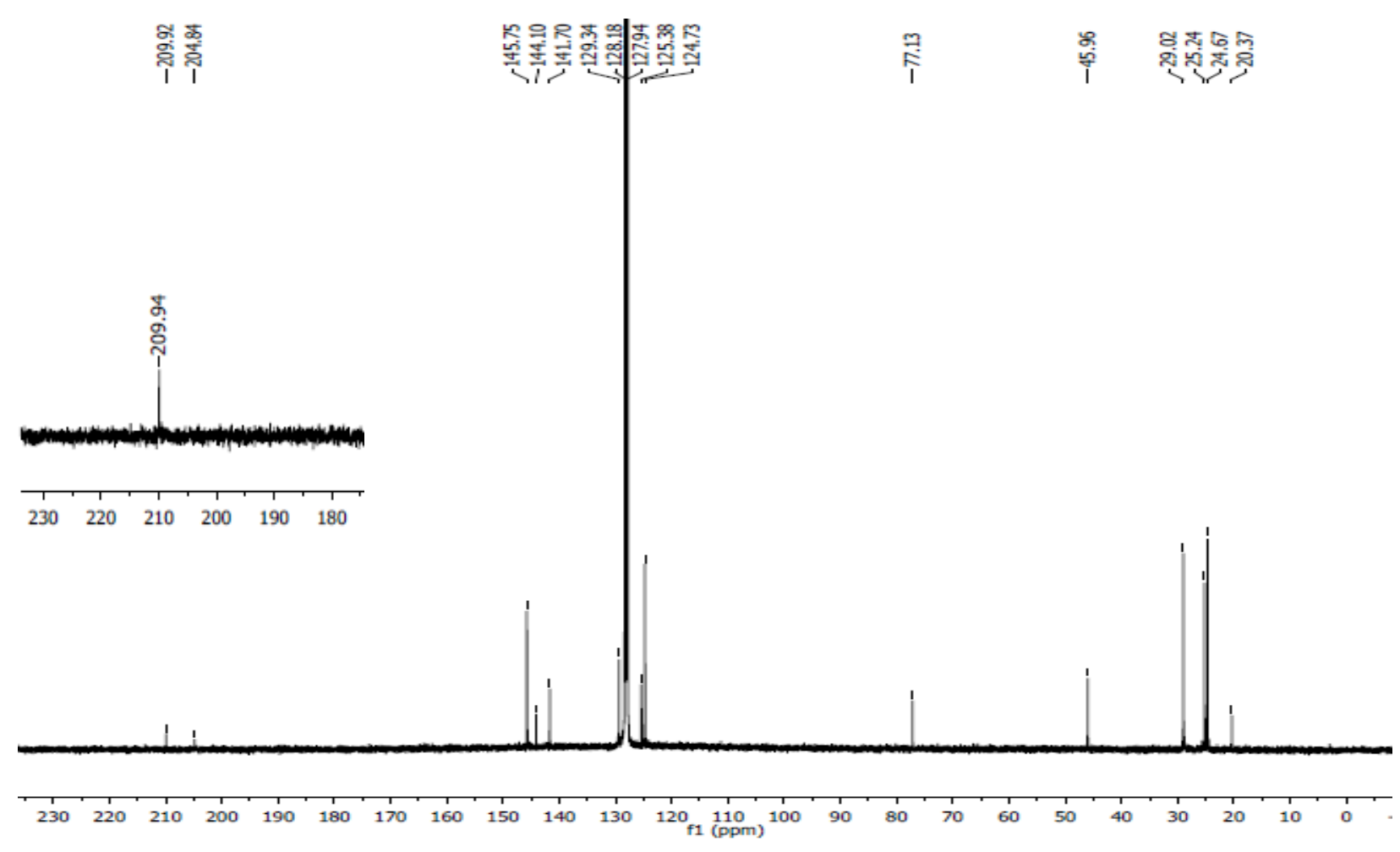

Figure S19. ${ }^{13} \mathrm{C}$ NMR spectrum of (6Dipp)copper(I) ( $N$-benzyl)formimidoyl overlaid with the DEPT-90 in $\mathrm{C}_{6} \mathrm{D}_{6}$. 


\section{Reaction of $[(5 \mathrm{Dipp}) \mathrm{CuH}]_{2}$ with 1-Hexene.}

Pinacolborane [4,4,5,5,- tetramethyl-1,3,2-dioxaborolane; HB(pin)] (0.055 mL, $0.037 \mathrm{mmol})$ was added to a solution of (5Dipp) $\mathrm{Cu}(\mathrm{O}-\mathrm{Bu})(\mathbf{1 b}, 0.020 \mathrm{~g}, 0.037 \mathrm{mmol})$ and 1.4-dimethoxybenzene (0.005 mg, $0.04 \mathrm{mmol}$; internal standard) in $\mathrm{C}_{6} \mathrm{D}_{6}(1 \mathrm{~mL})$ via syringe. The solution immediately became bright yellow in color and 1-hexene $(0.047 \mathrm{~mL}, 0.037 \mathrm{mmol})$ was then added via syringe. The solution was transferred to a J. Young NMR tube, and the reaction was monitored by ${ }^{1} \mathrm{H}$ NMR.

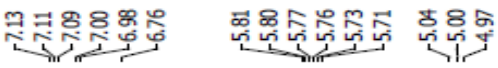

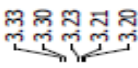

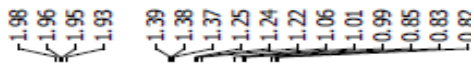

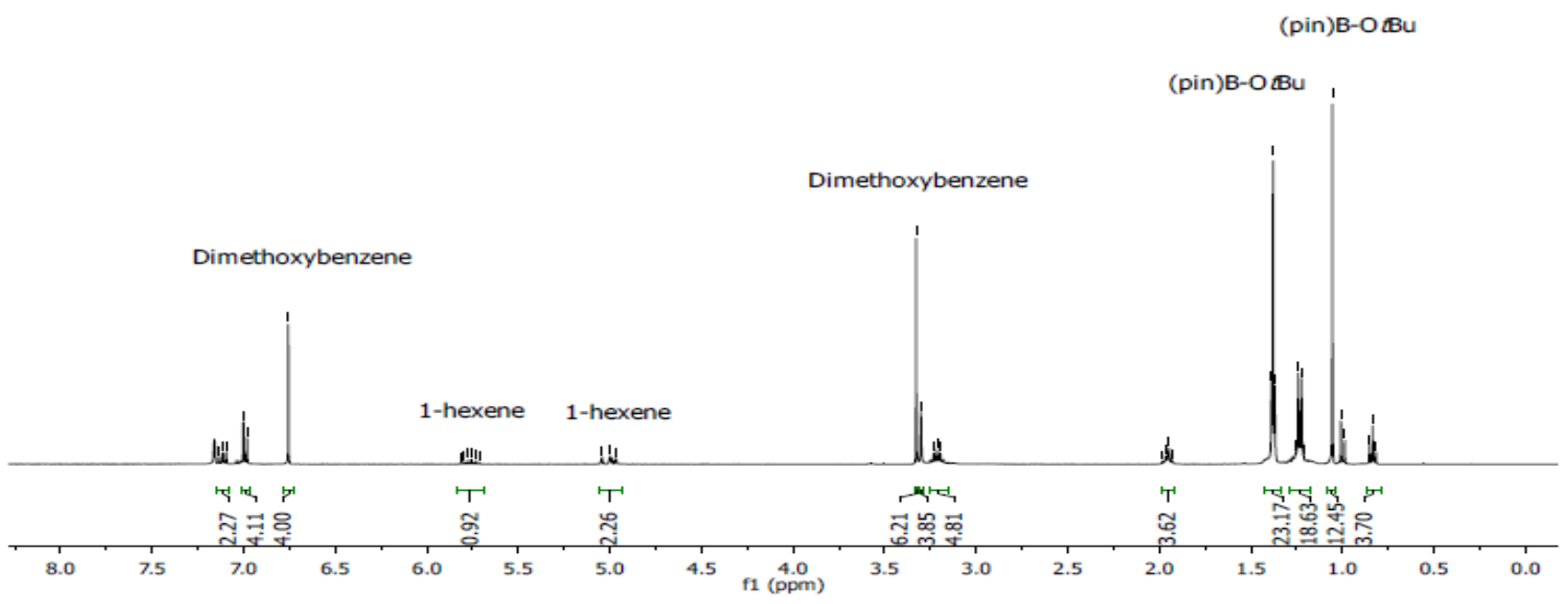

Figure S20. ${ }^{1} \mathrm{H}$ NMR spectrum of $[(5 \mathrm{Dipp}) \mathrm{CuH}]_{2}$ with 1 -hexene in $\mathrm{C}_{6} \mathrm{D}_{6}$ after $10 \mathrm{~min}$. 


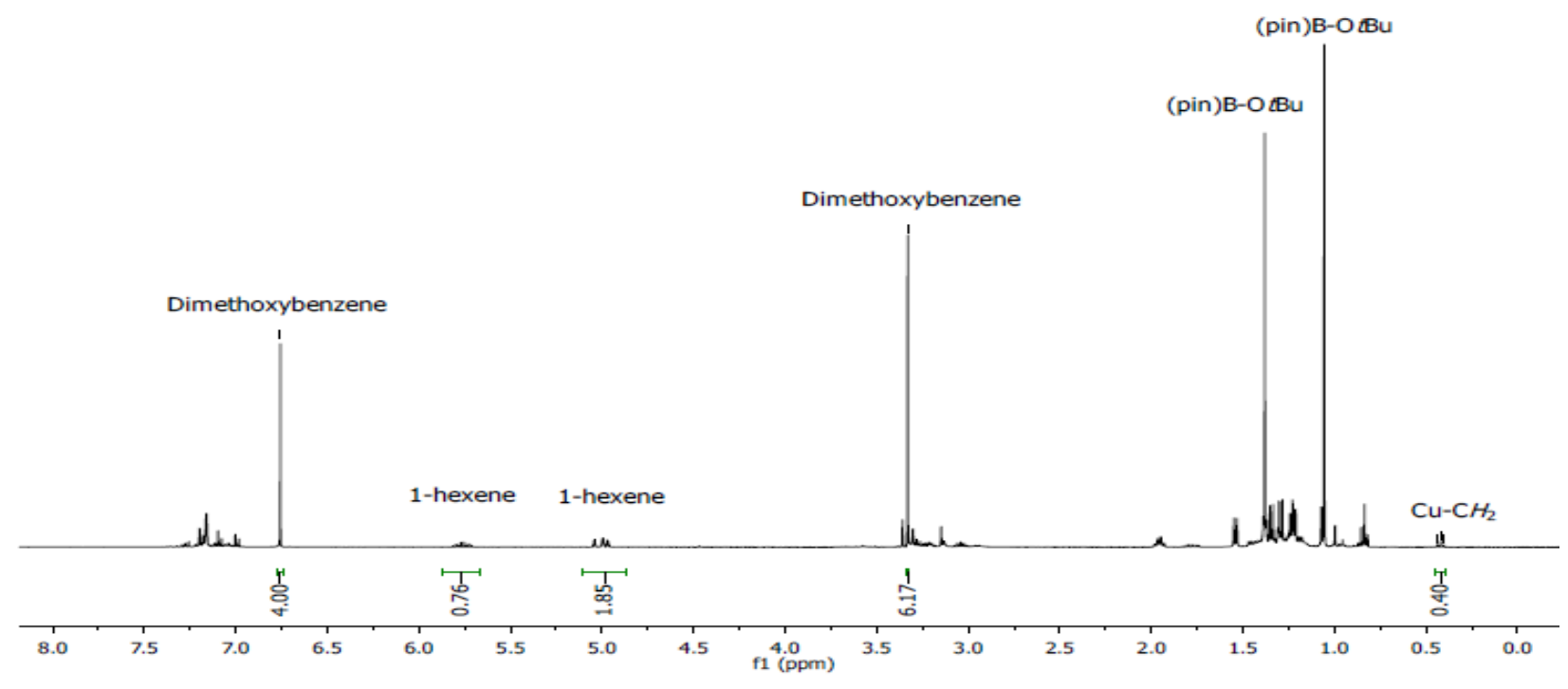

Figure S21. ${ }^{1} \mathrm{H}$ NMR spectrum of $[(5 \mathrm{Dipp}) \mathrm{CuH}]_{2}$ with 1 -hexene in $\mathrm{C}_{6} \mathrm{D}_{6}$ after $48 \mathrm{~h}$. 


\section{Reaction of [(7Dipp)CuH $]_{2}$ with benzyl isocyanide.}

Benzyl isocyanide $(0.027 \mathrm{~mL}, 0.023 \mathrm{mmol})$ was added to a solution of [(7Dipp)CuH $]_{2}(\mathbf{4 b}, 0.012 \mathrm{~g}$, $0.021 \mathrm{mmol})$ in $\mathrm{C}_{6} \mathrm{D}_{6}(0.7 \mathrm{~mL})$ via syringe. The solution was transferred to a J. Young NMR tube, and the reaction was monitored by ${ }^{1} \mathrm{H}$ NMR spectroscopy.

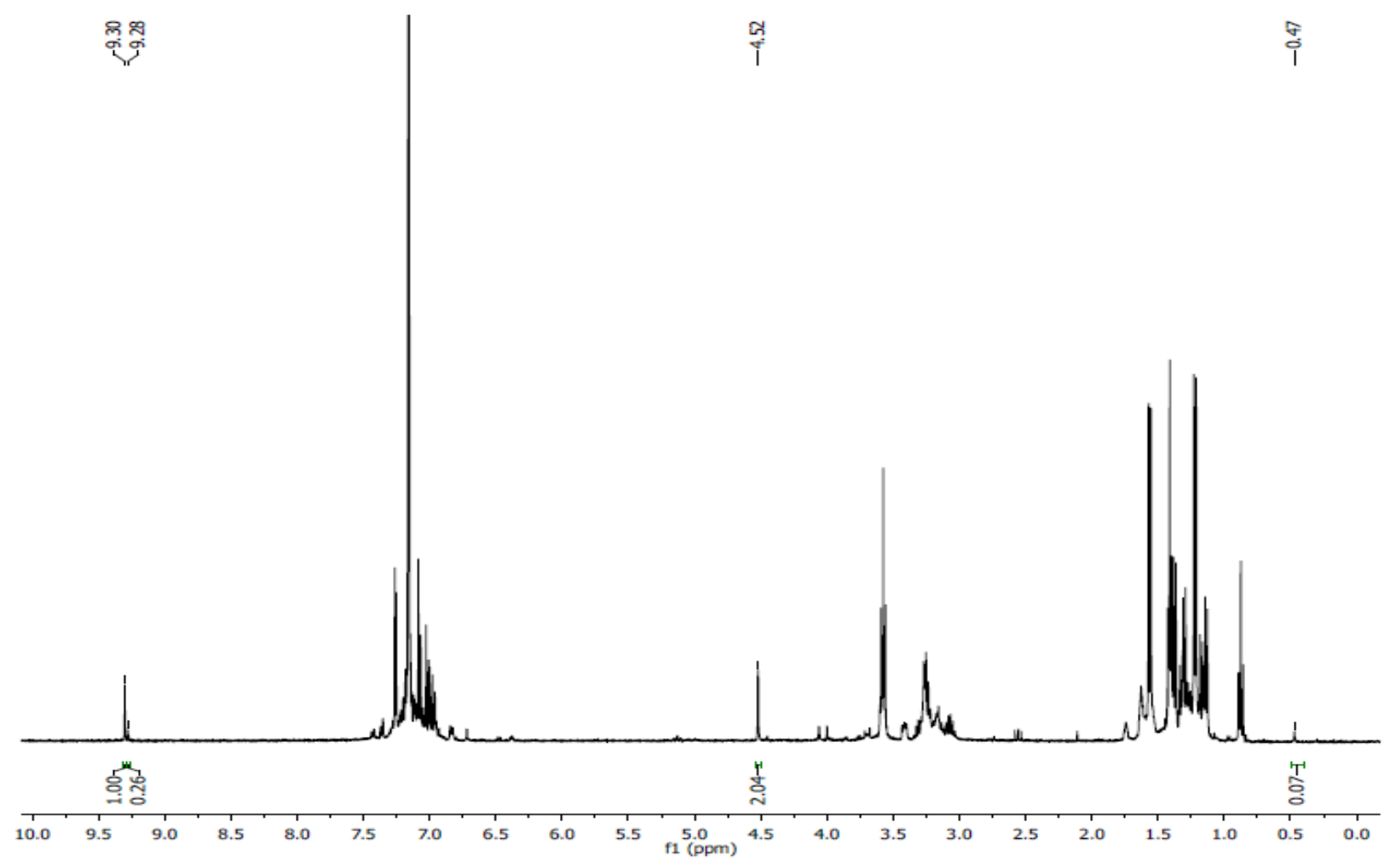

Figure S22. ${ }^{1} \mathrm{H}$ NMR spectrum of $[(7 \mathrm{Dipp}) \mathrm{CuH}]_{2}$ with benzyl isocyanide in $\mathrm{C}_{6} \mathrm{D}_{6}$ after $5.5 \mathrm{~h}$. 


\section{X-Ray Diffraction Studies}

\section{Compound $7 \mathbf{a}$}

Submitted by: Abraham Jordan

Georgia Institute of Technology

Solved by: John Bacsa

Sample ID: $\quad \mathbf{7 a}$

\section{Crystal Data and Experimental}

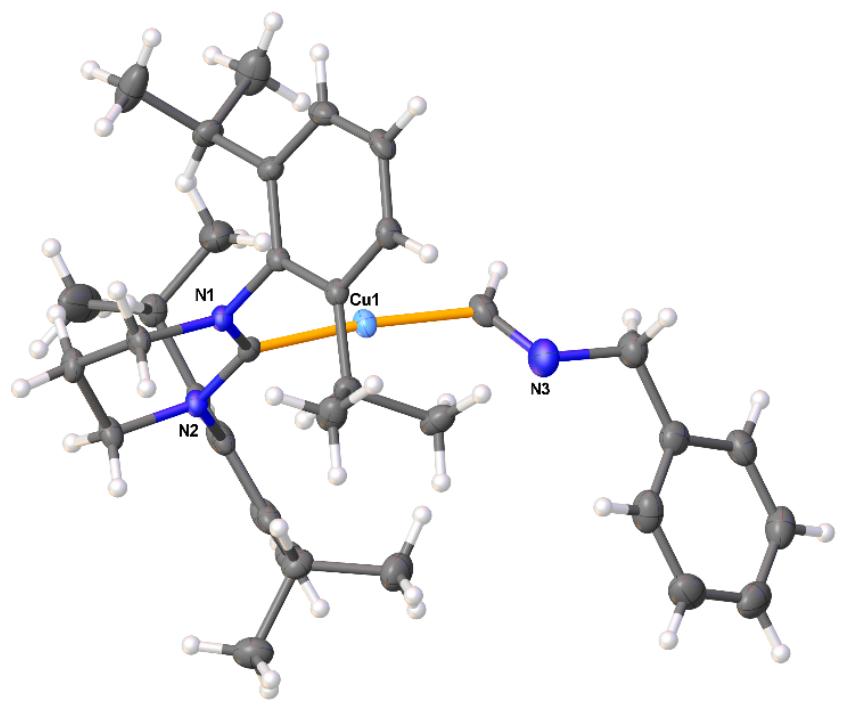

Figure S23: Thermal ellipsoid plot of 7a.

Experimental. Single yellow block-shaped crystals of 7a were recrystallised from a mixture of toluene and pentane by vapor diffusion. A suitable crystal $(0.64 \times 0.31 \times 0.22 \mathrm{~mm})$ was selected and mounted on a loop with paratone oil on a Bruker APEX-II CCD diffractometer. The crystal was cooled to $T=$ 100(2) K during the data collection. The structure was solved with ShelXT (Sheldrick, 2015) using direct and dual-space solution methods and by using Olex2 (Dolomanov et al., 2009) as the graphical interface. The model was refined with version of ShelXL-97 (Sheldrick, 2008) using Least Squares minimisation.

Crystal Data. $\mathrm{C}_{36} \mathrm{H}_{48} \mathrm{CuN}_{3}, M_{r}=586.31$, monoclinic, $\mathrm{P} 2{ }_{1} / \mathrm{c}$ (No. 14), $\mathrm{a}=$ 10.5381(13) $\AA, \quad b=19.114(2) \AA, \quad c=$ 16.3664(19) $\AA, \beta=97.200(2)^{\circ}, \alpha=\gamma=$ $90^{\circ}, V=3270.6(7) \AA^{3}, T=100(2) \mathrm{K}, Z=$ $4, \quad Z^{\prime}=1, \quad \mu\left(\mathrm{MoK}_{a}\right)=0.695,48654$ reflections measured, 9194 unique $\left(R_{\text {int }}=\right.$ 0.0424 ) which were used in all calculations. The final $w R_{2}$ was 0.1280 (all data) and $R_{l}$ was 0.0491 (I $>2(\mathrm{I})$ ). 
Table S1: Crystal data for compound 7a

\begin{tabular}{|c|c|}
\hline $\begin{array}{l}\text { CCDC Number } \\
\text { Formula }\end{array}$ & $\begin{array}{l}1446330 \\
\mathrm{C}_{36} \mathrm{H}_{48} \mathrm{CuN}_{3}\end{array}$ \\
\hline$D_{\text {calc. }} / \mathrm{g} \mathrm{cm}^{-3}$ & 1.191 \\
\hline$\mu / \mathrm{mm}^{-1}$ & 0.695 \\
\hline Formula Weight & 586.31 \\
\hline Colour & yellow \\
\hline Shape & block \\
\hline Max Size/mm & 0.64 \\
\hline Mid Size/mm & 0.31 \\
\hline Min Size/mm & 0.22 \\
\hline$T / \mathrm{K}$ & $100(2)$ \\
\hline Crystal System & monoclinic \\
\hline Space Group & $\mathrm{P} 2{ }_{1} / \mathrm{c}$ \\
\hline$a / \AA$ & $10.5381(13)$ \\
\hline$b / \AA$ & $19.114(2)$ \\
\hline$c / \AA$ & $16.3664(19)$ \\
\hline$\alpha l^{\circ}$ & 90 \\
\hline$\beta /^{\circ}$ & $97.200(2)$ \\
\hline$\gamma i^{\circ}$ & 90 \\
\hline $\mathrm{V} / \AA^{3}$ & $3270.6(7)$ \\
\hline$Z$ & 4 \\
\hline$Z^{\prime}$ & 1 \\
\hline$\Theta_{\min } j^{\circ}$ & 1.646 \\
\hline$\Theta_{\max } i^{\circ}$ & 29.632 \\
\hline Measured Refl. & 48654 \\
\hline Independent Refl. & 9194 \\
\hline Reflections Used & 7470 \\
\hline$R_{\text {int }}$ & 0.0424 \\
\hline Parameters & 369 \\
\hline Restraints & 0 \\
\hline Largest Peak & 1.075 \\
\hline Deepest Hole & -0.389 \\
\hline GooF & 1.062 \\
\hline$w R_{2}$ (all data) & 0.1280 \\
\hline$w R_{2}$ & 0.1180 \\
\hline$R_{l}$ (all data) & 0.0654 \\
\hline$R_{1}$ & 0.0491 \\
\hline
\end{tabular}


A yellow block-shaped crystal with dimensions $0.64 \times 0.31 \times 0.22 \mathrm{~mm}$ was mounted on a loop with paratone oil. Data were collected using a Bruker APEX-II CCD diffractometer equipped with an Oxford Cryosystems lowtemperature apparatus operating at $T=100(2) \mathrm{K}$.

Data were measured using $\phi$ and $\omega$ scans $1^{\circ}$ per frame for variable scan times using $\mathrm{MoK}_{a}$ radiation (sealed tube, $45 \mathrm{kV}, 35 \mathrm{~mA}$ ). The total number of runs and images was based on the strategy calculation from the program APEX2 (Bruker). The maximum resolution achieved was $\Theta=29.632^{\circ}$.

Unit cell indexing was performed by using the APEX2 (Bruker) software and refined using SAINT (Bruker, V8.34A, 2013) on 9967 reflections, 20\% of the observed reflections. Data reduction, scaling and absorption corrections were performed using SAINT (Bruker, V8.34A, 2013) and SADABS-2014/5 (Bruker,2014) was used for absorption correction. $w R_{2}$ (int) was 0.1026 before and 0.0469 after correction. The Ratio of minimum to maximum transmission is 0.8665 . The $\lambda / 2$ correction factor is 0.00150 . software. The final completeness is 99.90 out to 29.632 in $\Theta$. The absorption coefficient $(\mu)$ of this material is $0.695 \mathrm{~mm}^{-1}$ and the minimum and maximum transmissions are 0.6463 and 0.7459 .

The structure was solved with ShelXT (Sheldrick, 2015) using direct and dual-space solution methods and by using Olex2 (Dolomanov et al., 2009) as the graphical interface. The structure was refined by Least Squares using version of ShelXL-97 (Sheldrick, 2008). All non-hydrogen atoms were refined anisotropically.

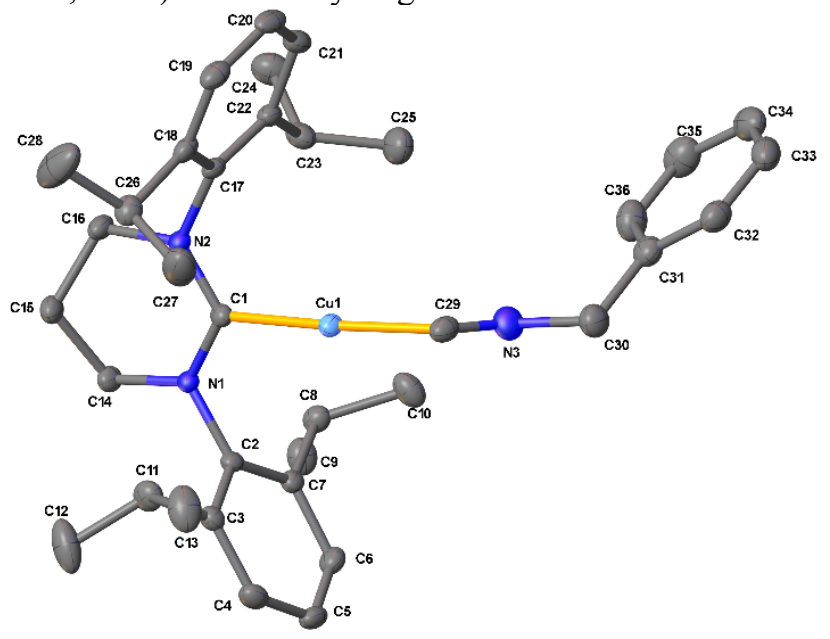

Figure S24: Thermal ellipsoid plot of 7a, hydrogen atoms are omitted for clarity. 


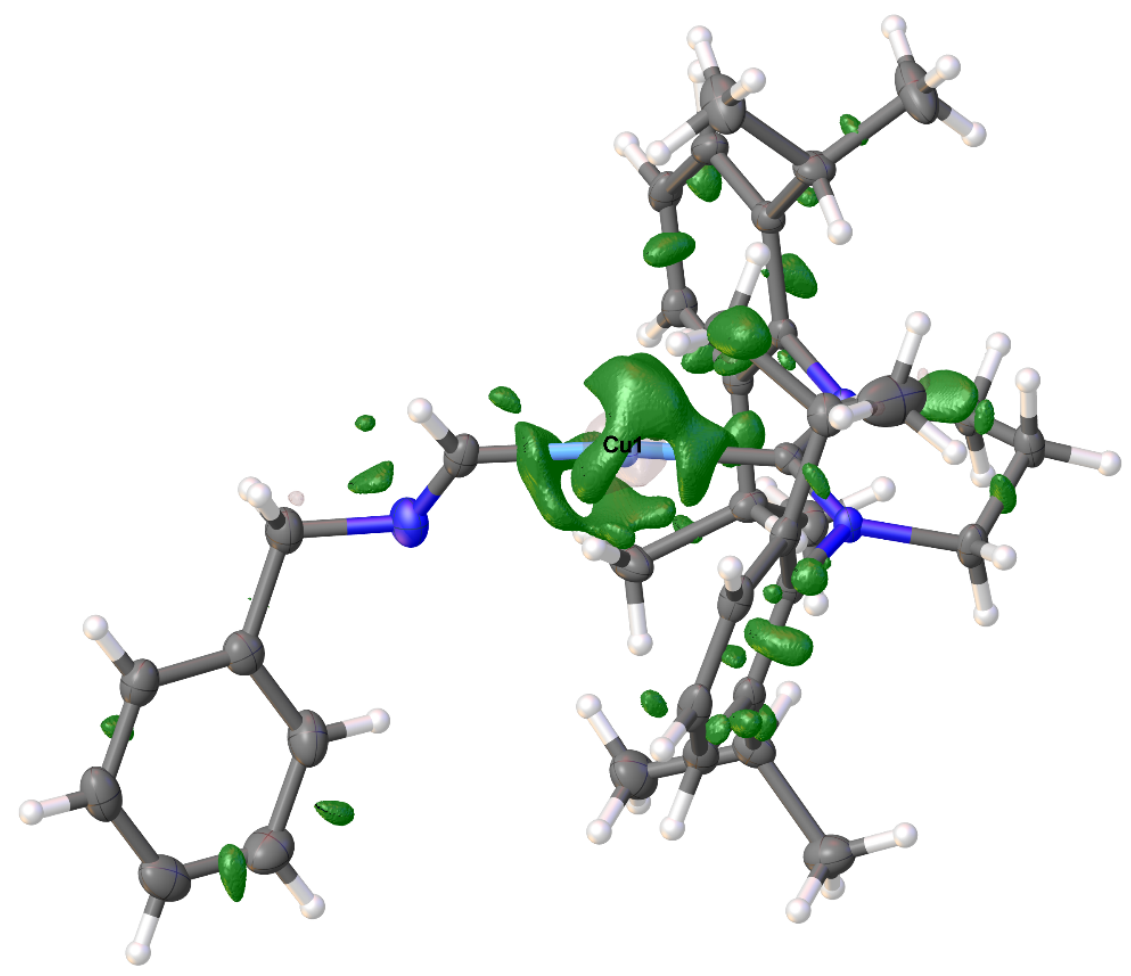

Figure S25: Plot of the residual electron density for $\mathbf{7 a}$.

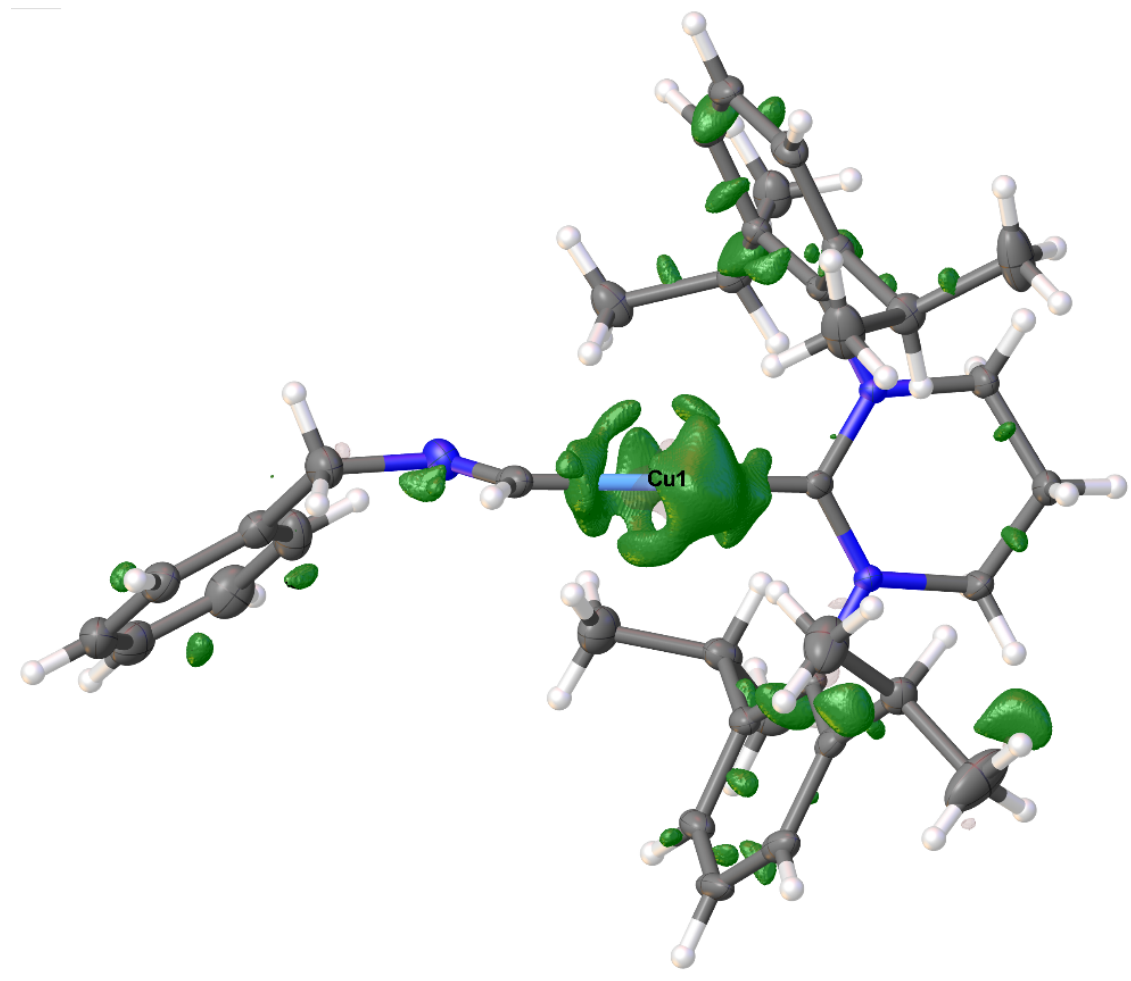

Figure S26: Plot of the residual electron density for $7 \mathbf{a}$. 


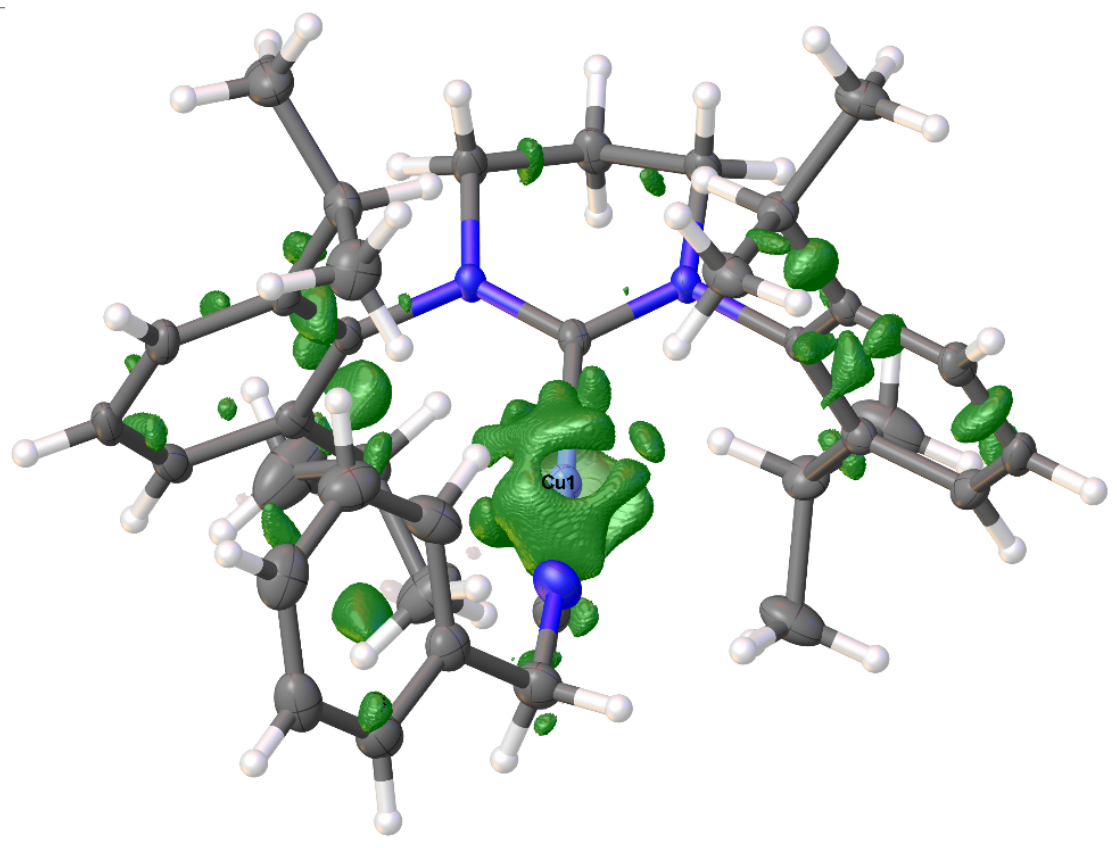

Figure S27: Plot of the residual electron density for 7a.
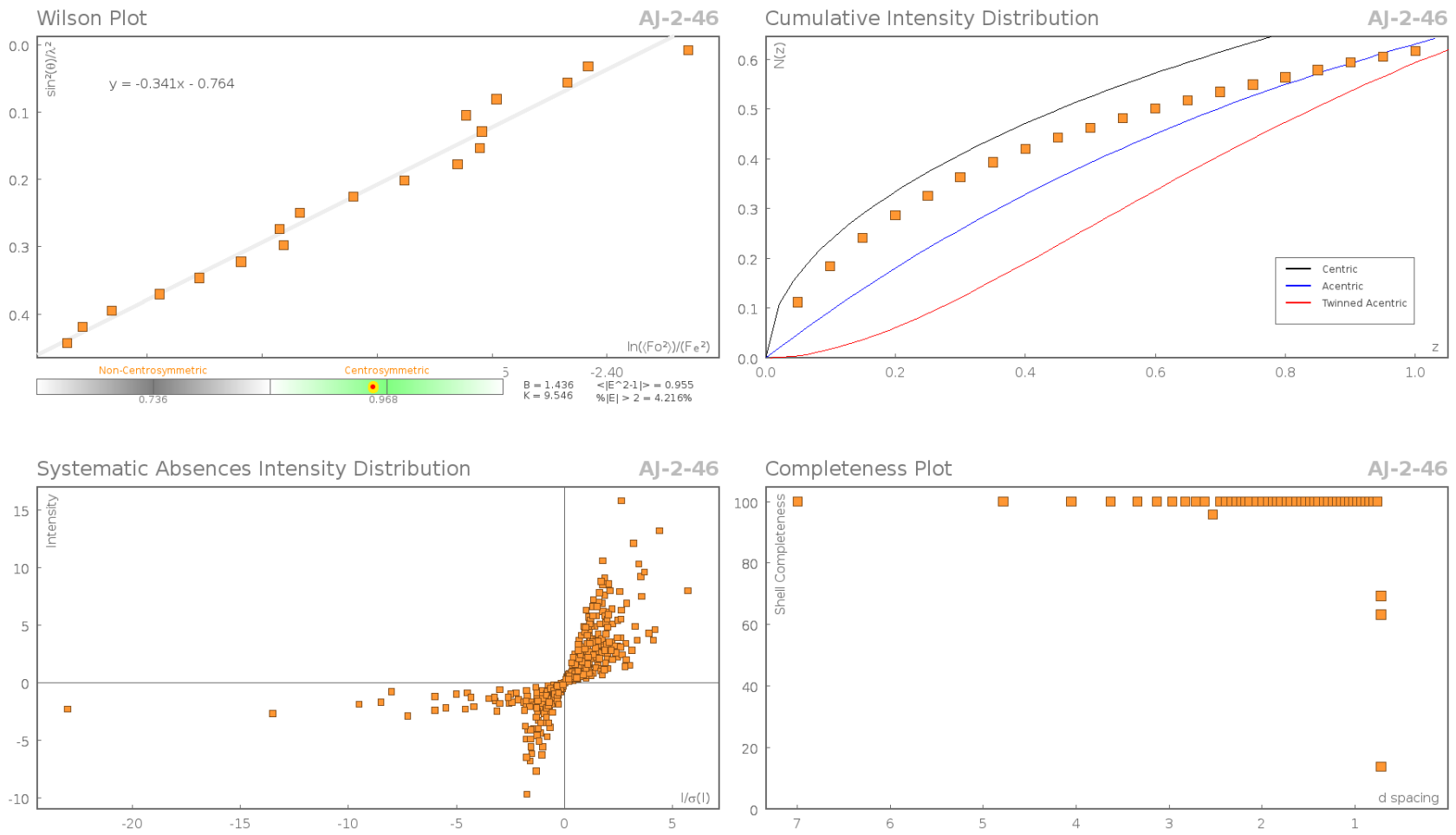

Figure S28: Data Plots: Diffraction Data 

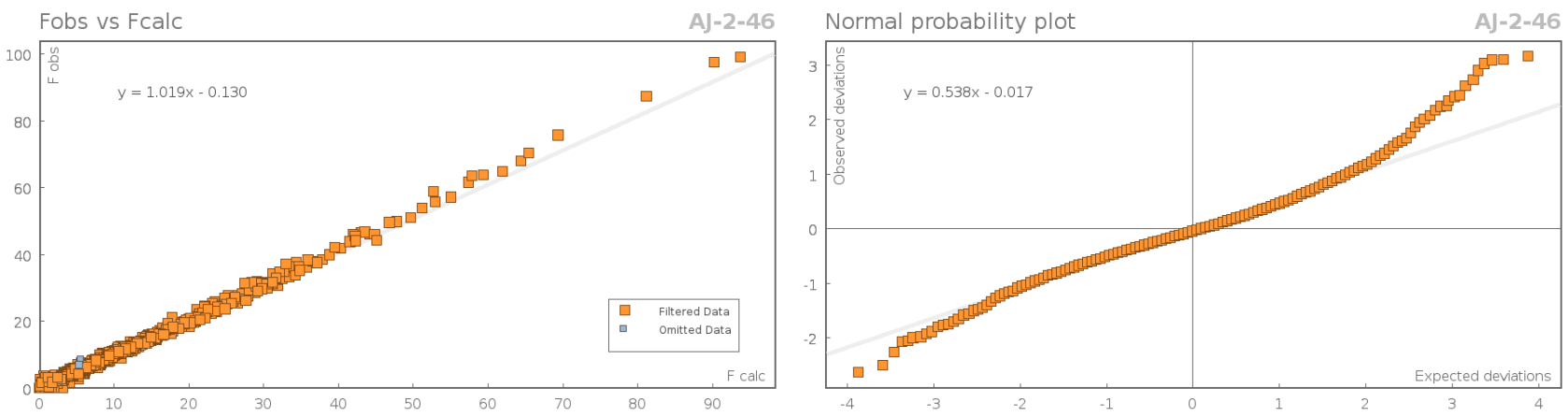

Figure S29: Data Plots: Refinement and Data

\section{Table S2: Reflection Statistics}

$\begin{array}{ll}\text { Total reflections (after filtering) } & 49467 \\ \text { Completeness } & 0.997 \\ \text { hklsub }>\text { max }</ \text { sub }>\text { collected } & (14,26,22) \\ \mathrm{hkl}_{\max } \text { used } & (14,26,22) \\ \text { Lim d } \mathrm{d}_{\max } \text { collected } & 100.0 \\ \mathrm{~d}_{\max } \text { used } & 16.24 \\ \text { Friedel pairs } & 11184 \\ \text { Inconsistent equivalents } & 0 \\ \mathrm{R}_{\text {sigma }} & 0.033 \\ \text { Omitted reflections } & 0 \\ \text { Multiplicity } & (14425,8742,4835,752,9) \\ \text { Removed systematic absences } & 801\end{array}$

$\begin{array}{ll}\text { Unique reflections } & 9194 \\ \text { Mean } \mathrm{I} / \sigma & 21.31 \\ \mathrm{hkl} \text { lsub }>\text { min }</ \text { sub }>\text { collected } & (-14,-26,-22) \\ \mathrm{hk}_{\min } \text { used } & (-14,0,0) \\ \text { Lim } \mathrm{d}_{\text {min }} \text { collected } & 0.36 \\ \mathrm{~d}_{\min } \text { used } & 0.72 \\ \text { Friedel pairs merged } & 1 \\ \mathrm{R}_{\text {int }} & 0.0424 \\ \text { Intensity transformed } & 0 \\ \text { Omitted by user (OMIT hkl) } & 12 \\ \text { Maximum mulitplicity } & 14 \\ \text { Filtered off (Shel/OMIT) } & 0\end{array}$
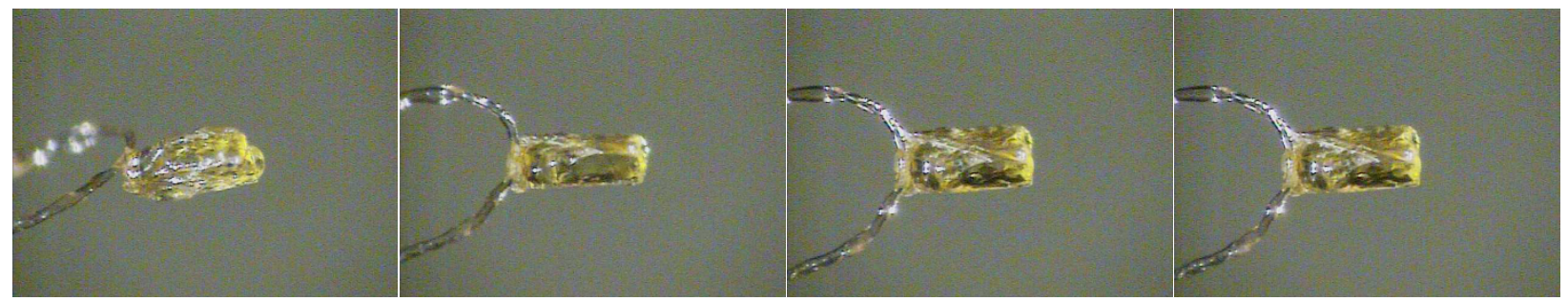

Figure S30: Images of the Crystal on the Diffractometer

Table S3: Fractional Atomic Coordinates $\left(\times 10^{4}\right)$ and Equivalent Isotropic Displacement Parameters $\left(\AA^{2} \times 10^{3}\right)$ for 7a. $U_{e q}$ is defined as $1 / 3$ of the trace of the orthogonalised $U_{i j}$.

\begin{tabular}{lllll}
\hline Atom & \multicolumn{1}{c}{$\mathbf{x}$} & $\mathbf{y}$ & $\mathbf{z}$ & \multicolumn{1}{c}{$\boldsymbol{U}_{\boldsymbol{e q}}$} \\
\hline Cu1 & $5023.7(2)$ & $2941.4(2)$ & $4237.8(2)$ & $16.73(7)$ \\
N1 & $5839.6(14)$ & $1833.5(8)$ & $5348.8(9)$ & $14.4(3)$ \\
N2 & $5646.2(15)$ & $2882.4(8)$ & $5996.3(10)$ & $15.5(3)$ \\
N3 & $3070.1(19)$ & $3249.6(10)$ & $2965.7(12)$ & $29.6(4)$ \\
C18 & $6367.4(19)$ & $4097.1(10)$ & $5973.5(11)$ & $19.2(4)$ \\
C3 & $6910.9(17)$ & $1378(1)$ & $4213.1(12)$ & $17.2(3)$ \\
C23 & $2986.1(19)$ & $3314.3(11)$ & $5977.5(13)$ & $23.3(4)$ \\
C4 & $6851(2)$ & $975.4(11)$ & $3500.6(12)$ & $22.0(4)$ \\
C8 & $3424.4(18)$ & $1201.9(11)$ & $4671.0(12)$ & $19.7(4)$ \\
C21 & $3848(2)$ & $4546.4(11)$ & $6023.2(12)$ & $22.7(4)$
\end{tabular}




\begin{tabular}{|c|c|c|c|c|}
\hline Atom & $\mathbf{x}$ & $\mathbf{y}$ & $\mathbf{z}$ & $U_{e q}$ \\
\hline $\mathrm{C} 6$ & $4639.0(19)$ & $717.9(11)$ & $3557.9(12)$ & $20.8(4)$ \\
\hline $\mathrm{C} 15$ & $6762(2)$ & $1936.8(11)$ & $6792.2(13)$ & $22.4(4)$ \\
\hline $\mathrm{C} 26$ & 7718.2(19) & $3851.3(11)$ & $5907.9(13)$ & $22.9(4)$ \\
\hline $\mathrm{C} 5$ & $5730(2)$ & $647.8(11)$ & $3173.0(12)$ & $22.2(4)$ \\
\hline $\mathrm{C} 16$ & $5937(2)$ & $2579.0(11)$ & $6828.0(12)$ & $21.6(4)$ \\
\hline C19 & $6075(2)$ & $4808.3(11)$ & $6003.0(12)$ & $23.3(4)$ \\
\hline $\mathrm{C} 20$ & $4827(2)$ & $5027.9(11)$ & $6030.9(13)$ & $25.3(4)$ \\
\hline $\mathrm{C} 14$ & $6159.2(19)$ & $1446.2(10)$ & $6127.2(12)$ & $19.5(4)$ \\
\hline $\mathrm{C} 11$ & $8145.8(18)$ & $1743.1(12)$ & $4551.4(13)$ & $23.6(4)$ \\
\hline $\mathrm{C} 29$ & $4266(2)$ & $3348.3(11)$ & $3222.2(12)$ & $23.1(4)$ \\
\hline $\mathrm{C} 30$ & $2509(2)$ & $3609.4(12)$ & 2214.1(14) & $28.7(5)$ \\
\hline C31 & $1242(2)$ & $3949.7(12)$ & $2315.3(14)$ & $26.1(4)$ \\
\hline C34 & $-1112(2)$ & $4581.8(14)$ & $2478.3(16)$ & $36.0(5)$ \\
\hline C9 & 2894(2) & 496.6(12) & $4906.4(14)$ & $27.5(4)$ \\
\hline $\mathrm{C} 10$ & $2424(2)$ & $1613.1(12)$ & $4099.5(15)$ & $27.6(5)$ \\
\hline $\mathrm{C} 32$ & $801(2)$ & $4520.5(12)$ & $1832.2(14)$ & $28.4(5)$ \\
\hline $\mathrm{C} 35$ & $-685(2)$ & $4020.8(15)$ & $2968.9(16)$ & $37.1(6)$ \\
\hline $\mathrm{C} 24$ & $2369(2)$ & $3354.4(13)$ & $6774.2(16)$ & $32.6(5)$ \\
\hline $\mathrm{C} 25$ & $2007(2)$ & $3429.4(14)$ & $5214.1(16)$ & $35.7(5)$ \\
\hline $\mathrm{C} 33$ & $-362(2)$ & $4828.2(13)$ & $1914.2(15)$ & $34.3(5)$ \\
\hline $\mathrm{C} 2$ & $5792.2(17)$ & $1436.9(9)$ & $4595.8(11)$ & $14.3(3)$ \\
\hline $\mathrm{C} 22$ & $4085.8(18)$ & $3828.7(10)$ & $5993.0(11)$ & $18.3(4)$ \\
\hline $\mathrm{C} 17$ & $5354.1(18)$ & $3619.3(10)$ & $5970.7(11)$ & $16.4(3)$ \\
\hline $\mathrm{C} 1$ & $5585.6(16)$ & $2519.6(9)$ & $5294.5(11)$ & $14.5(3)$ \\
\hline $\mathrm{C} 7$ & $4648.5(18)$ & $1115.2(10)$ & $4277.5(11)$ & $15.9(3)$ \\
\hline $\mathrm{C} 36$ & $477(2)$ & $3702.8(14)$ & $2888.8(15)$ & $34.2(5)$ \\
\hline $\mathrm{C} 12$ & $9133(2)$ & $1220.5(15)$ & 4936.4(19) & $43.9(7)$ \\
\hline $\mathrm{C} 13$ & $8688(2)$ & $2178.4(14)$ & $3895.7(17)$ & $37.8(6)$ \\
\hline $\mathrm{C} 27$ & $8016(2)$ & $3886.6(17)$ & $5028.5(16)$ & $42.5(6)$ \\
\hline $\mathrm{C} 28$ & $8729(3)$ & $4249(2)$ & $6472(2)$ & $57.8(9)$ \\
\hline
\end{tabular}

Table S4: Anisotropic Displacement Parameters $\left(\times 10^{4}\right)$ for 7a. The anisotropic displacement factor exponent takes the form: $-2 \pi^{2}\left[h^{2} a^{*^{2}} \times U_{11}+\ldots+2 h k a * \times b^{*} \times U_{12}\right]$

\begin{tabular}{llllrrr}
\hline Atom & \multicolumn{1}{c}{$\boldsymbol{U}_{\mathbf{1 1}}$} & \multicolumn{1}{c}{$\boldsymbol{U}_{\mathbf{2 2}}$} & \multicolumn{1}{c}{$\boldsymbol{U}_{33}$} & $\boldsymbol{U}_{23}$ & $\boldsymbol{U}_{\mathbf{1 3}}$ & $\boldsymbol{U}_{\mathbf{1 2}}$ \\
\hline Cu1 & $22.14(12)$ & $12.94(12)$ & $14.58(12)$ & $-0.03(8)$ & $0.21(8)$ & $1.05(8)$ \\
N1 & $16.5(7)$ & $11.8(7)$ & $14.1(7)$ & $-1.1(6)$ & $-0.8(5)$ & $0.9(5)$ \\
N2 & $19.0(7)$ & $12.8(7)$ & $14.1(7)$ & $-1.3(6)$ & $-0.9(6)$ & $0.8(5)$ \\
N3 & $35.8(10)$ & $23.1(9)$ & $28.7(10)$ & $4.7(8)$ & $-0.5(8)$ & $2.5(8)$ \\
C18 & $25.7(9)$ & $19.0(9)$ & $12.0(8)$ & $-1.4(7)$ & $-0.6(7)$ & $-2.8(7)$ \\
C3 & $16.5(8)$ & $15.5(9)$ & $19.5(9)$ & $1.3(7)$ & $2.5(7)$ & $1.7(6)$ \\
C23 & $21.9(9)$ & $17.9(10)$ & $29.9(11)$ & $-2.4(8)$ & $2.4(8)$ & $2.0(7)$ \\
C4 & $24.7(9)$ & $22.1(10)$ & $20.4(9)$ & $0.9(8)$ & $7.2(7)$ & $6.0(7)$ \\
C8 & $17.1(8)$ & $22(1)$ & $20.3(9)$ & $-2.0(7)$ & $3.1(7)$ & $-2.6(7)$ \\
C21 & $30.8(10)$ & $17.5(9)$ & $19.7(9)$ & $-1.1(7)$ & $2.7(8)$ & $7.2(8)$ \\
C6 & $23.6(9)$ & $18.7(10)$ & $18.8(9)$ & $-2.7(7)$ & $-2.1(7)$ & $-2.4(7)$ \\
C15 & $27.3(10)$ & $18.9(10)$ & $19.0(9)$ & $1.0(7)$ & $-4.4(7)$ & $3.2(7)$ \\
C26 & $23.5(9)$ & $20.2(10)$ & $24.1(10)$ & $0.9(8)$ & $-0.3(8)$ & $-4.2(7)$ \\
C5 & $33.5(11)$ & $18(1)$ & $14.7(9)$ & $-3.0(7)$ & $1.8(8)$ & $4.1(8)$ \\
C16 & $32.2(10)$ & $16.8(9)$ & $14.6(9)$ & $-1.6(7)$ & $-1.7(7)$ & $2.2(7)$ \\
C19 & $36.0(11)$ & $16.6(9)$ & $16.7(9)$ & $-0.5(7)$ & $1.3(8)$ & $-6.1(8)$ \\
C20 & $46.1(12)$ & $11.9(9)$ & $17.5(9)$ & $-0.4(7)$ & $2.8(8)$ & $4.1(8)$ \\
C14 & $26.2(9)$ & $15.8(9)$ & $15.8(8)$ & $2.4(7)$ & $0.3(7)$ & $2.6(7)$ \\
C11 & $15.5(8)$ & $28.4(11)$ & $27.3(10)$ & $-0.9(8)$ & $3.9(7)$ & $-1.4(7)$ \\
C29 & $34.3(11)$ & $17(1)$ & $18.0(9)$ & $-0.5(7)$ & $2.7(8)$ & $0.9(8)$ \\
C30 & $33.1(11)$ & $27.0(11)$ & $25.5(11)$ & $4.0(9)$ & $1.2(9)$ & $2.7(9)$ \\
C31 & $26.5(10)$ & $25.2(11)$ & $25.3(10)$ & $-3.5(8)$ & $-1.2(8)$ & $-2.5(8)$ \\
& & & & &
\end{tabular}




\begin{tabular}{lllcccc}
\hline Atom & \multicolumn{1}{c}{$\boldsymbol{U}_{11}$} & $\boldsymbol{U}_{22}$ & $\boldsymbol{U}_{33}$ & $\boldsymbol{U}_{23}$ & $\boldsymbol{U}_{13}$ & $\boldsymbol{U}_{12}$ \\
\hline C34 & $25.7(11)$ & $42.0(15)$ & $38.1(13)$ & $-12.2(11)$ & $-4.6(9)$ & $1.2(10)$ \\
C9 & $24.5(10)$ & $28.4(11)$ & $29.1(11)$ & $8.5(9)$ & $2.3(8)$ & $-4.3(8)$ \\
C10 & $19.6(9)$ & $25.8(11)$ & $38.1(12)$ & $7.3(9)$ & $6.4(8)$ & $1.8(8)$ \\
C32 & $36.0(12)$ & $27.2(11)$ & $21.4(10)$ & $0.1(8)$ & $1.7(9)$ & $-1.6(9)$ \\
C35 & $29.2(11)$ & $48.6(16)$ & $34.2(13)$ & $-1.0(11)$ & $6.1(10)$ & $-9.1(10)$ \\
C24 & $31.9(11)$ & $29.6(12)$ & $38.6(13)$ & $0.2(10)$ & $13(1)$ & $-0.3(9)$ \\
C25 & $27.7(11)$ & $41.0(14)$ & $36.0(13)$ & $-4.3(11)$ & $-4.7(9)$ & $0.8(10)$ \\
C33 & $39.8(13)$ & $27.9(12)$ & $31.8(12)$ & $-1.8(10)$ & $-8.8(10)$ & $4.5(10)$ \\
C2 & $18.2(8)$ & $10.4(8)$ & $14.3(8)$ & $-0.4(6)$ & $1.6(6)$ & $1.7(6)$ \\
C22 & $24.0(9)$ & $15.7(9)$ & $14.7(8)$ & $-2.0(7)$ & $0.4(7)$ & $2.9(7)$ \\
C17 & $24.4(9)$ & $12.4(8)$ & $12.0(8)$ & $-2.1(6)$ & $0.1(7)$ & $0.4(7)$ \\
C1 & $12.8(7)$ & $13.5(8)$ & $16.7(8)$ & $-1.5(6)$ & $-0.5(6)$ & $-0.9(6)$ \\
C7 & $19.0(8)$ & $12.1(8)$ & $16.1(8)$ & $0.2(6)$ & $0.1(6)$ & $1.1(6)$ \\
C36 & $35.5(12)$ & $32.3(13)$ & $32.8(12)$ & $9(1)$ & $-3.8(10)$ & $-6.1(10)$ \\
C12 & $24.4(11)$ & $46.9(16)$ & $56.8(17)$ & $26.3(13)$ & $-9.4(11)$ & $-5.8(10)$ \\
C13 & $31.6(12)$ & $33.6(13)$ & $46.1(15)$ & $15.0(11)$ & $-3.1(10)$ & $-10.7(10)$ \\
C27 & $31.2(12)$ & $64.4(19)$ & $32.2(13)$ & $-2.7(12)$ & $5(1)$ & $13.7(12)$ \\
C28 & $29.7(13)$ & $97(3)$ & $45.8(17)$ & $-30.8(17)$ & $1.1(12)$ & $-12.4(15)$
\end{tabular}

Table S5: Bond Lengths in $\AA$ for $7 \mathbf{a}$.

\begin{tabular}{lll}
\hline Atom & Atom & Length $/ \AA$ \\
\hline $\mathrm{Cu} 1$ & $\mathrm{C} 29$ & $1.916(2)$ \\
$\mathrm{Cu} 1$ & $\mathrm{C} 1$ & $1.9326(18)$ \\
$\mathrm{N} 1$ & $\mathrm{C} 14$ & $1.475(2)$ \\
$\mathrm{N} 1$ & $\mathrm{C} 2$ & $1.442(2)$ \\
$\mathrm{N} 1$ & $\mathrm{C} 1$ & $1.339(2)$ \\
$\mathrm{N} 2$ & $\mathrm{C} 16$ & $1.476(2)$ \\
$\mathrm{N} 2$ & $\mathrm{C} 17$ & $1.441(2)$ \\
$\mathrm{N} 2$ & $\mathrm{C} 1$ & $1.336(2)$ \\
$\mathrm{N} 3$ & $\mathrm{C} 29$ & $1.292(3)$ \\
$\mathrm{N} 3$ & $\mathrm{C} 30$ & $1.467(3)$ \\
$\mathrm{C} 18$ & $\mathrm{C} 26$ & $1.516(3)$ \\
$\mathrm{C} 18$ & $\mathrm{C} 19$ & $1.396(3)$ \\
$\mathrm{C} 18$ & $\mathrm{C} 17$ & $1.405(3)$ \\
$\mathrm{C} 3$ & $\mathrm{C} 4$ & $1.392(3)$ \\
$\mathrm{C} 3$ & $\mathrm{C} 11$ & $1.519(3)$ \\
$\mathrm{C} 3$ & $\mathrm{C} 2$ & $1.407(2)$ \\
$\mathrm{C} 23$ & $\mathrm{C} 24$ & $1.530(3)$ \\
$\mathrm{C} 23$ & $\mathrm{C} 25$ & $1.533(3)$ \\
$\mathrm{C} 23$ & $\mathrm{C} 22$ & $1.518(3)$ \\
$\mathrm{C} 4$ & $\mathrm{C} 5$ & $1.384(3)$ \\
$\mathrm{C} 8$ & $\mathrm{C} 9$ & $1.527(3)$ \\
$\mathrm{C} 8$ & $\mathrm{C} 10$ & $1.535(3)$ \\
\end{tabular}

\begin{tabular}{lll}
\hline Atom & Atom & Length/ $\mathbf{\AA}$ \\
\hline C8 & C7 & $1.521(3)$ \\
C21 & C20 & $1.381(3)$ \\
C21 & C22 & $1.396(3)$ \\
C6 & C5 & $1.385(3)$ \\
C6 & C7 & $1.400(3)$ \\
C15 & C16 & $1.509(3)$ \\
C15 & C14 & $1.515(3)$ \\
C26 & C27 & $1.513(3)$ \\
C26 & C28 & $1.523(3)$ \\
C19 & C20 & $1.387(3)$ \\
C11 & C12 & $1.521(3)$ \\
C11 & C13 & $1.525(3)$ \\
C30 & C31 & $1.513(3)$ \\
C31 & C32 & $1.393(3)$ \\
C31 & C36 & $1.394(3)$ \\
C34 & C35 & $1.381(4)$ \\
C34 & C33 & $1.372(4)$ \\
C32 & C33 & $1.381(3)$ \\
C35 & C36 & $1.388(4)$ \\
C2 & C7 & $1.395(3)$ \\
C22 & C17 & $1.400(3)$ \\
& &
\end{tabular}

Table S6: Bond Angles in ${ }^{\circ}$ for $7 \mathbf{a}$.

\begin{tabular}{llll}
\hline Atom & Atom & Atom & \multicolumn{1}{c}{ Angle/ } \\
\hline $\mathrm{C} 29$ & $\mathrm{Cu} 1$ & $\mathrm{C} 1$ & $173.28(8)$ \\
$\mathrm{C} 2$ & $\mathrm{~N} 1$ & $\mathrm{C} 14$ & $116.99(15)$ \\
$\mathrm{C} 1$ & $\mathrm{~N} 1$ & $\mathrm{C} 14$ & $124.81(15)$ \\
$\mathrm{C} 1$ & $\mathrm{~N} 1$ & $\mathrm{C} 2$ & $118.21(15)$ \\
$\mathrm{C} 17$ & $\mathrm{~N} 2$ & $\mathrm{C} 16$ & $115.45(15)$ \\
$\mathrm{C} 1$ & $\mathrm{~N} 2$ & $\mathrm{C} 16$ & $124.81(16)$ \\
$\mathrm{C} 1$ & $\mathrm{~N} 2$ & $\mathrm{C} 17$ & $119.68(15)$
\end{tabular}

\begin{tabular}{lllc}
\hline Atom & Atom & Atom & Angle $^{\circ}$ \\
\hline C29 & N3 & C30 & $118.57(19)$ \\
C19 & C18 & C26 & $121.19(18)$ \\
C19 & C18 & C17 & $117.52(18)$ \\
C17 & C18 & C26 & $121.24(18)$ \\
C4 & C3 & C11 & $120.40(17)$ \\
C4 & C3 & C2 & $117.69(17)$ \\
C2 & C3 & C11 & $121.90(17)$
\end{tabular}




\begin{tabular}{|c|c|c|c|}
\hline Atom & Atom & Atom & Angle $/^{\circ}$ \\
\hline$\overline{\mathrm{C} 24}$ & $\mathrm{C} 23$ & $\mathrm{C} 25$ & $111.89(19)$ \\
\hline $\mathrm{C} 22$ & $\mathrm{C} 23$ & $\mathrm{C} 24$ & $111.00(18)$ \\
\hline $\mathrm{C} 22$ & $\mathrm{C} 23$ & $\mathrm{C} 25$ & $110.80(18)$ \\
\hline C5 & $\mathrm{C} 4$ & $\mathrm{C} 3$ & $121.42(18)$ \\
\hline C9 & C8 & $\mathrm{C} 10$ & $111.15(17)$ \\
\hline $\mathrm{C} 7$ & C8 & C9 & $111.53(17)$ \\
\hline C7 & C8 & $\mathrm{C} 10$ & $110.59(16)$ \\
\hline $\mathrm{C} 20$ & C21 & $\mathrm{C} 22$ & $121.17(19)$ \\
\hline C5 & $\mathrm{C} 6$ & $\mathrm{C} 7$ & $121.06(18)$ \\
\hline $\mathrm{C} 16$ & $\mathrm{C} 15$ & C14 & $109.87(16)$ \\
\hline $\mathrm{C} 18$ & $\mathrm{C} 26$ & $\mathrm{C} 28$ & $113.2(2)$ \\
\hline $\mathrm{C} 27$ & $\mathrm{C} 26$ & $\mathrm{C} 18$ & $111.25(18)$ \\
\hline $\mathrm{C} 27$ & $\mathrm{C} 26$ & $\mathrm{C} 28$ & $110.0(2)$ \\
\hline $\mathrm{C} 4$ & $\mathrm{C} 5$ & C6 & $119.80(18)$ \\
\hline N2 & $\mathrm{C} 16$ & C15 & $109.75(16)$ \\
\hline $\mathrm{C} 20$ & $\mathrm{C} 19$ & $\mathrm{C} 18$ & $120.69(19)$ \\
\hline $\mathrm{C} 21$ & $\mathrm{C} 20$ & C19 & $120.56(19)$ \\
\hline N1 & $\mathrm{C} 14$ & $\mathrm{C} 15$ & $109.85(16)$ \\
\hline $\mathrm{C} 3$ & $\mathrm{C} 11$ & $\mathrm{C} 12$ & $111.08(19)$ \\
\hline $\mathrm{C} 3$ & $\mathrm{C} 11$ & $\mathrm{C} 13$ & $111.96(18)$ \\
\hline $\mathrm{C} 12$ & C11 & $\mathrm{C} 13$ & $110.65(19)$ \\
\hline N3 & C29 & $\mathrm{Cu} 1$ & $120.40(16)$ \\
\hline N3 & C30 & $\mathrm{C} 31$ & $112.21(19)$ \\
\hline C32 & C31 & C30 & $120.7(2)$ \\
\hline C32 & C31 & C36 & $117.9(2)$ \\
\hline C36 & C31 & $\mathrm{C} 30$ & $121.4(2)$ \\
\hline $\mathrm{C} 33$ & C34 & C35 & $119.0(2)$ \\
\hline $\mathrm{C} 33$ & C32 & C31 & $121.0(2)$ \\
\hline C34 & C35 & C36 & $120.8(2)$ \\
\hline C34 & $\mathrm{C} 33$ & C32 & $120.8(2)$ \\
\hline C3 & $\mathrm{C} 2$ & N1 & $118.76(16)$ \\
\hline C7 & $\mathrm{C} 2$ & N1 & 119.13(15) \\
\hline C7 & $\mathrm{C} 2$ & C3 & $122.11(17)$ \\
\hline $\mathrm{C} 21$ & $\mathrm{C} 22$ & $\mathrm{C} 23$ & $119.80(18)$ \\
\hline $\mathrm{C} 21$ & $\mathrm{C} 22$ & $\mathrm{C} 17$ & $117.26(18)$ \\
\hline $\mathrm{C} 17$ & $\mathrm{C} 22$ & $\mathrm{C} 23$ & $122.94(17)$ \\
\hline $\mathrm{C} 18$ & $\mathrm{C} 17$ & N2 & $118.42(17)$ \\
\hline $\mathrm{C} 22$ & $\mathrm{C} 17$ & N2 & $118.67(17)$ \\
\hline $\mathrm{C} 22$ & $\mathrm{C} 17$ & $\mathrm{C} 18$ & $122.81(18)$ \\
\hline N1 & $\mathrm{C} 1$ & $\mathrm{Cu} 1$ & $120.27(13)$ \\
\hline N2 & $\mathrm{C} 1$ & $\mathrm{Cu} 1$ & $121.94(13)$ \\
\hline $\mathrm{N} 2$ & $\mathrm{C} 1$ & N1 & 117.61(16) \\
\hline C6 & $\mathrm{C} 7$ & $\mathrm{C} 8$ & $119.87(17)$ \\
\hline $\mathrm{C} 2$ & $\mathrm{C} 7$ & C8 & $122.19(16)$ \\
\hline $\mathrm{C} 2$ & C7 & C6 & 117.91(17) \\
\hline C35 & C36 & C31 & $120.5(2)$ \\
\hline
\end{tabular}


Table S7: Hydrogen Fractional Atomic Coordinates $\left(\times 10^{4}\right)$ and Equivalent Isotropic Displacement Parameters $\left(\AA^{2} \times 10^{3}\right)$ for 7 a. $U_{e q}$ is defined as $1 / 3$ of the trace of the orthogonalised $U_{i j}$.

\begin{tabular}{|c|c|c|c|c|}
\hline Atom & & & & $U_{e q}$ \\
\hline$\overline{\mathrm{H} 23}$ & 3338 & 2842 & 5941 & 28 \\
\hline H4 & 7580 & 925 & 3239 & 26 \\
\hline H8 & 3631 & 1475 & 5177 & 24 \\
\hline H21 & 3016 & 4703 & 6038 & 27 \\
\hline H6 & 3888 & 497 & 3335 & 25 \\
\hline $\mathrm{H} 15 \mathrm{~A}$ & 6851 & 1700 & 7321 & 27 \\
\hline H15B & 7607 & 2073 & 6675 & 27 \\
\hline H26 & 7771 & 3359 & 6076 & 27 \\
\hline H5 & 5710 & 382 & 2696 & 27 \\
\hline H16A & 5148 & 2454 & 7040 & 26 \\
\hline H16B & 6382 & 2921 & 7197 & 26 \\
\hline H19 & 6724 & 5138 & 6004 & 28 \\
\hline $\mathrm{H} 20$ & 4648 & 5503 & 6055 & 30 \\
\hline H14A & 6749 & 1070 & 6048 & 23 \\
\hline H14B & 5390 & 1242 & 6294 & 23 \\
\hline H11 & 7951 & 2063 & 4987 & 28 \\
\hline H29 & 4763 & 3618 & 2912 & 28 \\
\hline $\mathrm{H} 30 \mathrm{~A}$ & 2384 & 3275 & 1765 & 34 \\
\hline H30B & 3100 & 3964 & 2070 & 34 \\
\hline H34 & -1896 & 4790 & 2530 & 43 \\
\hline H9A & 2139 & 570 & 5168 & 41 \\
\hline H9B & 3525 & 258 & 5280 & 41 \\
\hline $\mathrm{H} 9 \mathrm{C}$ & 2685 & 218 & 4420 & 41 \\
\hline H10A & 2166 & 1344 & 3612 & 41 \\
\hline H10B & 2788 & 2048 & 3950 & 41 \\
\hline $\mathrm{H} 10 \mathrm{C}$ & 1693 & 1704 & 4379 & 41 \\
\hline H32 & 1297 & 4697 & 1448 & 34 \\
\hline H35 & -1183 & 3854 & 3358 & 45 \\
\hline $\mathrm{H} 24 \mathrm{~A}$ & 2031 & 3816 & 6833 & 49 \\
\hline $\mathrm{H} 24 \mathrm{~B}$ & 3000 & 3255 & 7235 & 49 \\
\hline $\mathrm{H} 24 \mathrm{C}$ & 1689 & 3018 & 6755 & 49 \\
\hline $\mathrm{H} 25 \mathrm{~A}$ & 1363 & 3072 & 5188 & 53 \\
\hline $\mathrm{H} 25 \mathrm{~B}$ & 2431 & 3408 & 4728 & 53 \\
\hline $\mathrm{H} 25 \mathrm{C}$ & 1614 & 3880 & 5247 & 53 \\
\hline H33 & -640 & 5207 & 1583 & 41 \\
\hline H36 & 747 & 3322 & 3220 & 41 \\
\hline $\mathrm{H} 12 \mathrm{~A}$ & 8777 & 954 & 5350 & 66 \\
\hline H12B & 9877 & 1467 & 5184 & 66 \\
\hline $\mathrm{H} 12 \mathrm{C}$ & 9368 & 911 & 4518 & 66 \\
\hline H13A & 8935 & 1875 & 3476 & 57 \\
\hline H13B & 9422 & 2434 & 4143 & 57 \\
\hline $\mathrm{H} 13 \mathrm{C}$ & 8049 & 2501 & 3655 & 57 \\
\hline $\mathrm{H} 27 \mathrm{~A}$ & 7370 & 3639 & 4676 & 64 \\
\hline $\mathrm{H} 27 \mathrm{~B}$ & 8835 & 3676 & 4994 & 64 \\
\hline $\mathrm{H} 27 \mathrm{C}$ & 8033 & 4367 & 4858 & 64 \\
\hline H28A & 8715 & 4734 & 6316 & 87 \\
\hline $\mathrm{H} 28 \mathrm{~B}$ & 9558 & 4056 & 6426 & 87 \\
\hline $\mathrm{H} 28 \mathrm{C}$ & 8551 & 4209 & 7032 & 87 \\
\hline
\end{tabular}




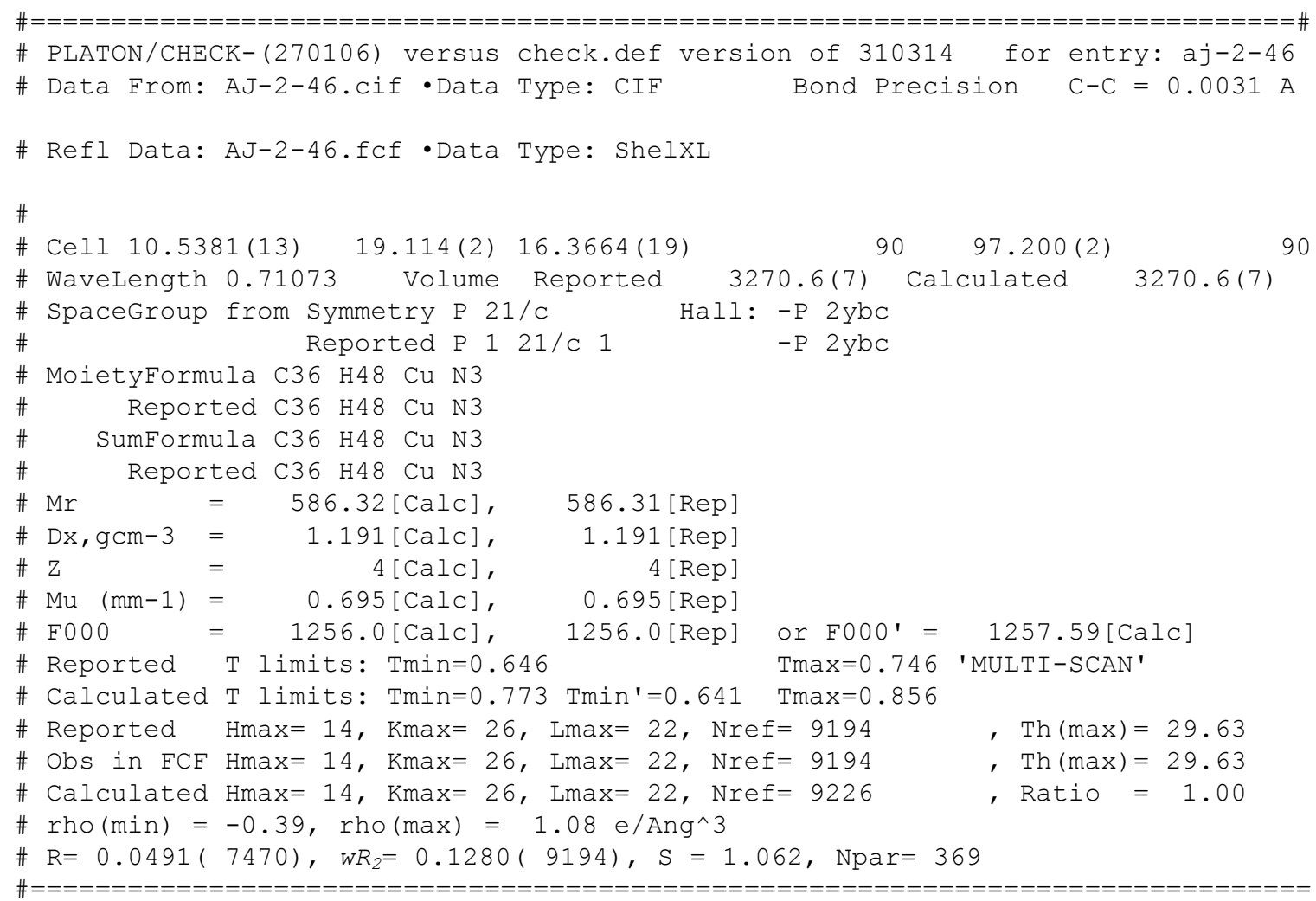

>>> The Following ALERTS were generated $<<<$

number_ALERT_alert-type_alert-level text
Format: alert-

\begin{tabular}{|c|c|c|}
\hline \multirow{2}{*}{\multicolumn{3}{|c|}{ 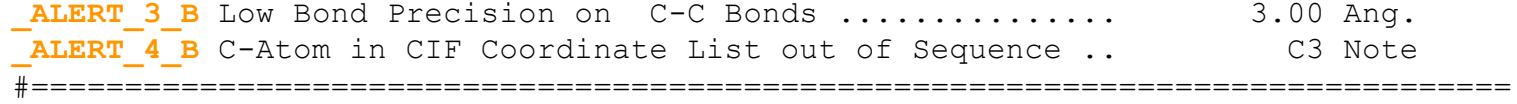 }} \\
\hline & & \\
\hline \multicolumn{3}{|c|}{ 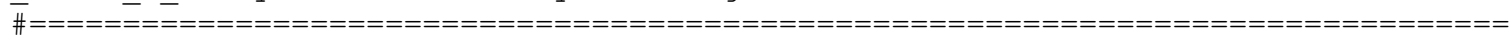 } \\
\hline \\
\hline ALERT_2-C & Ratio of Maximum / Minimum Residual Density .... & 2.76 Why ? \\
\hline ALERT 2 G & $U_{e q}(\max ) / U_{e q}(\min )$ Range & 4.04 Ratio \\
\hline ALERT_2-G & Hirshfeld Test Diff $(\mathrm{M}-\mathrm{X})$ Cul $\quad-\mathrm{C} 29 \quad \ldots$ & 5.77 \\
\hline ALERT_2_G & $U_{e q}$ as Compared to Neighbors for ..... & C26 Chec \\
\hline ALERT_ 4 G & CIF Input Record(s) with more than 80 Characters & ! \\
\hline ALERT_3_G & FCF Refl Between THmin \& STh/L= 0.600 & 3 Why ? \\
\hline ALERT_4_G & of FCF Reflections Above STh/L= 0.600 & 30 Note \\
\hline
\end{tabular}

ALERT_Level and ALERT_Type Summary

2 ALERT Level A = In General: Serious Problem

1 ALERT_Level_B = Potentially Serious Problem

8 ALERT_Level_C $=$ Check \& Explain

4 ALERT Type 2 Indicator that the Structure Model may be Wrong or Deficient.

2 ALERT_Type_3 Indicator that the structure Quality may be Low.

5 ALERT Type_4 Improvement, Methodology, Query or Suggestion. 


\section{References}

(1) Kuhn, K. M.; Grubbs, R. H. Org. Lett. 2008, 10, 2075.

(2) Laitar, D. S., Synthetic and Catalytic Studies of Group 11 N-Heterocyclic Carbene

Complexes. Dissertation, Massachusetts Institute of Technology. Cambridge Massachusetts, 2006.

(3) Kolychev, E. L.; Portnyagin, I. A.; Shuntikov, V. V.; Khrustalev, V. N.; Nechaev, M. S. J. Organomet. Chem. 2009, 694, 2454.

(4) Fluegge, S.; Anoop, A.; Goddard, R.; Thiel, W.; Fürstner, A. Chem. - Eur. J. 2009, 15, 8558.

(5) Yoo, W.-J.; Nguyen, T. V. Q.; Kobayashi, S. Angew. Chem. Int. Ed. 2014, 53, 10213.

(6) APEX2 suite for crystallographic software, Bruker AXS, Madison, WI (2014).

(7) Dolomanov, O. V.; Bourhis L. J.; Gildea, R. J.; and. Howard, J. A. K.; Puschmann, H. J. Appl. Cryst., 2009, 42, 339-341.

(8) SAINT-8.34A-2013 • Software for the Integration of CCD Detector System Bruker Analytical X-ray Systems, Bruker AXS, Madison, WI (2013).

(9) Sheldrick, G.M. Acta Cryst., 2008, A64, 339-341.

(10) Sheldrick, G.M. Acta Cryst., 2014, A71, 3-8. 